\title{
Acellular dermal matrices: Use in reconstructive and aesthetic breast surgery
}

\author{
Sheina A Macadam MD MHS, Peter A Lennox MD
}

SA Macadam, PA Lennox. Acellular dermal matrices: Use in reconstructive and aesthetic breast surgery. Can J Plast Surg 2012;20(2):75-90.

Acellular dermal matrices (ADMs) were first described for use in breast surgery in 2001. Since this initial report, ADMs have become an increasingly common component of implant-based breast procedures. ADMs have shown promise for use in both aesthetic and reconstructive breast surgery; however, concerns about their use remain because of the significant costs associated with these products. The present article reviews the history of ADM use in breast surgery and the outcomes reported to date. Common techniques for placement of ADMs in aesthetic revisionary and breast reconstruction surgery are provided, and use in the setting of chest wall irradiation and capsular contracture is discussed. Finally, the authors comment on the cost implications of these products in the Canadian and American health care systems.

Key Words: Acelluar dermal matrices; Breast aesthetic surgery; Breast reconstruction; Economic analysis

$\Delta$ cellular dermal matrices (ADMs) were initially described for use

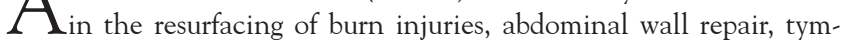
panic membrane replacement, dural repairs and gingival grafting $(1-8)$. In the field of breast surgery, ADMs were first introduced for use in revisional aesthetic surgery, including correction of implant rippling, symmastia and soft tissue deficits $(9,10)$. In 2005, Breuing and Warren (11) were the first to report on the use of ADMs in reconstructive breast surgery. They reported on a series of 10 patients who underwent bilateral mastectomies and direct-to-implant (single-stage) alloplastic reconstruction using a sling of AlloDerm (LifeCell Corporation, USA) inferiorly. These authors stated that their technique "avoids or shortens the tissue expansion/implant reconstructive process, avoids mastectomy flap contraction during the latency period of expansion, provides an additional layer of tissue between the skin and the implant, and offers an additional option for immediate, singlestage breast implant reconstruction". The advantages of conversion of traditional two-stage reconstruction to a single-stage surgery in the appropriate patient were soon recognized and this approach has become standard of care in many surgical centres.

Many surgeons also use ADMs in a two-stage approach using a tissue expander. This technique was first described by Bindingnavele et al (12) in 2007. They believed that advantages included decreased postoperative pain, faster expansion, elimination of the need for elevation of the serratus anterior muscle for lateral coverage of the prosthesis, improved lower pole projection, better aesthetic shape and improved soft tissue characteristics. The addition of ADMs to the traditional twostage tissue expansion reconstruction has become common; however, this application may be limited by cost in many centres.

This evolution has resulted in the incorporation of ADMs into more than $60 \%$ of all alloplastic reconstructions in the United States in less than one decade (13). AlloDerm was the first ADM to be described in the literature and is human derived (ie, allogenic).

\author{
Les matrices dermiques acellulaires : leur \\ utilisation en chirurgie mammaire reconstructive et \\ esthétique
}

Les matrices dermiques acellulaires (MDA) ont d'abord été décrites dans le cadre d'une utilisation en chirurgie mammaire en 2001. Depuis ce premier rapport, l'utilisation des MDA se généralise pour les interventions d'implants mammaires. Les MDA sont prometteuses en chirurgie mammaire esthétique et reconstructive, mais il reste des préoccupations quant à leur utilisation en raison à leur coût très élevé. Le présent article permet d'analyser l'évolution de l'utilisation des MDA en chirurgie mammaire et les issues déclarées jusqu'à maintenant. Les techniques courantes d'implantation des MDA dans le cadre de chirurgies mammaires de révision esthétique et de reconstruction sont présentées, et leur utilisation en irradiation de la paroi thoracique et de la contracture capsulaire est exposée. Enfin, les auteurs commentent les répercussions sur les coûts de ces produits dans le système de santé canadien et américain.

Subsequently, multiple ADMs have entered the market derived from both allogenic and xenogenic donor sources. AlloDerm has the longest history and is most referenced in the literature, but other products include Flex HD (Ethicon, USA), DermaMatrix (Synthes, USA), AlloMax (Bard Davol, USA) and SurgiMend (TEI Biosciences, USA). Non-ADM synthetic mesh products have also been introduced and these include TiMesh (Biomet, USA) (titanized) and TIGR Matrix (Novus Scientific, USA) (absorbable synthetic) mesh. These products differ in their processing and, as a result, have differences in handling, incorporation, shelf life and cost. Presumably, many of these variables may affect outcomes; however, to date, differences in outcomes have not been established in the literature.

Over the past decade, growth in the ADM market has been explosive. This has been due to increased surgeon and patient awareness, perceived benefits and reports of favourable outcomes. However, potential advantages need to be assessed in terms of cost in today's economic environment. Additionally, well-designed studies are required to assess true outcomes and to compare these outcomes with those of non-ADM reconstruction.

\section{SCIENTIFIC REVIEW}

An optimal ADM should possess collagen and extracellular matrix components, and should not be recognized as foreign. Ideally, the ADM would be recognized as host tissue so as to induce minimal or no host immune inflammatory response. The ADM should be repopulated by host cells and ultimately revascularized and incorporated into the host tissue. The commercially available ADMs undergo several processing steps with proprietary differences. Processing includes mechanical separation of tissues, decellularization, disinfection, and dehydration or lyophilization (freeze drying) in some cases. Finally, some products are terminally sterilized. Each of these steps can affect the architecture of the ADM and, ultimately, interaction with host tissue (14). 


\section{Decellularization}

This step is required for all ADMs. Antigenic epitopes associated with cells must be removed to avoid a host-mediated immune inflammatory reaction or rejection. In contrast, molecules that comprise the extracellular matrix are preserved across species and, are therefore, tolerated. Decellularization uses detergents, and different detergents will have varying effects depending on the donor tissue.

\section{Dehydration}

ADMs can be dehydrated either through lyophilization or by vacuum pressing. Advantages of dehydration include ease of handling, potential improvement in shelf life and reduced loss of intrinsic growth factors. Disadvantages include reduction of the thickness of the scaffold and more compaction of fibres. Dehydrated tissues generally cannot resorb as much water as was present before dehydration; this is believed to be due to disruption in the glycosaminoglycan component of the tissues (15).

\section{Sterilization}

In general, terminal sterilization has been shown to have a negative effect on the mechanical properties of a scaffold. Ethylene oxide, gamma radiation and electron beam irradiation have all been shown to affect the strength of matrices. There is no clear evidence in the literature that one type of terminal sterilization offers advantages or better outcomes. AlloMax, SurgiMend and AlloDerm RTU are terminally sterilized, whereas most others are aseptically processed.

\section{Incorporation}

The three main immunological responses to an implanted ADM are integration, resorption and encapsulation. Integration involves the lack of immunological recognition, rapid revascularization and incorporation, and is the desired response. Resorption results in an inflammatory reaction at the ADM/host interface, with eventual breakdown and elimination of the ADM (16). Encapsulation is a result of a foreign body reaction with scar tissue surrounding the ADM. Sandor et al (17) reported that encapsulation is more common with cross-linked matrices. Although there have been no studies reporting specifically on histology within the breast, integration is the desired outcome to avoid an inflammatory reaction around the breast prosthesis.

\section{REVIEW OF TECHNIQUES USED IN BREAST SURGERY}

The first report of ADM use in breast surgery was published in 2001. In this series involving 34 patients, Duncan (9) used AlloDerm in the correction of rippling after breast implant surgery. This author's technique involved suturing the ADM along the obliquely oriented inferior pectoralis muscle and to the lateral and inferior chest wall. In patients with superomedial pectoral muscle disruption or absence, a patch of allograft was sutured along the undersurface of the defect after capsular elevation. Increased lateral tissue coverage and support helped to camouflage visible rippling in this series of patients. In patients with superomedial pectoral muscle disruption or absence, a patch of allograft was sutured along the undersurface of the defect after capsular elevation. In 2003, a small case series published by Baxter (10) further expanded the use of ADMs in aesthetic breast surgery to include correction of symmastia, soft tissue augmentation for atrophy, capsular reinforcement and placement of ADM into the breast after capsulotomy for capsular contracture.

Shortly after the introduction of ADMs into the field of revisional aesthetic breast surgery, the benefits were recognized and applied to reconstructive surgery. Presently, ADMs are used routinely in many centres in both primary and revisional alloplastic breast reconstructive and aesthetic surgery. Applications include the following:

- Expansion of the submuscular pocket to allow for direct-to-implant breast reconstruction;

- Expansion of the submuscular pocket to enhance two-stage breast reconstruction;
- Correction of symmastia;

- Incorporation into the upper pole to camouflage surface irregularities and rippling;

- Correction of inframammary fold malposition and 'bottoming out'; and

- Provision of an interface when performing capsulotomies or capsulectomy for recurrent capsular contracture.

A review of these techniques is presented below.

\section{Use in direct-to-implant reconstruction}

The goal of direct-to-implant reconstruction is to create a naturalappearing breast mound in a single stage without compromising mastectomy flap viability. The ideal patient characteristics include a small to medium nonptotic breast, good skin elasticity and a planned small to medium prosthetic reconstruction as well as maximal skin sparing mastectomy techniques (Figures $1 \mathrm{~A}$ and $1 \mathrm{~B}$ ). Advantages include elimination of the second stage of traditional tissue expander/implant reconstruction, rapid return to work and expedited administration of adjuvant therapy if required. Additionally, precise positioning of the implant and inferior support may decrease the number of revision surgeries required. Direct-to-implant reconstruction is not suitable in patients with a very large breast skin envelope and is controversial in patients requiring adjuvant radiotherapy or with a history of chest wall irradiation. Excessively thin mastectomy flaps are considered a contraindication to direct-to-implant reconstruction because the volume of the permanent implant may produce additional tension on the closure and lead to mastectomy flap necrosis.

Techniques vary slightly from author to author; however, direct-toimplant reconstruction typically involves suturing a $6 \mathrm{~cm} \times 16 \mathrm{~cm}$ piece of ADM to the inferior pectoralis muscle after its origin has been released from the chest wall, draping the ADM over the inferior surface of the chosen implant and suturing the ADM to the inferior and lateral chest wall (Figures 2A and 2B). After completion of the skinsparing or nipple-sparing mastectomy, the inferior origin of the pectoralis muscle is identified. This is released using electrocautery from its lateral edge to the inferior sternum in a manner commonly used in subpectoral aesthetic breast augmentation (Figure 3). A subpectoral plane is then developed to release the pectoralis muscle from the chest wall. While this is taking place, the ADM is prepared according to specific manufacturer's instructions. For AlloDerm, this involves soaking the ADM in a two-step warm saline bath for $10 \mathrm{~min}$ to $40 \mathrm{~min}$ with gentle agitation. This product should be used within $4 \mathrm{~h}$ of rehydration (18). FlexHD, DermaMatrix, AlloMax, Strattice (LifeCell Corporation, USA) and SurgiMend have similar instructions for use, depending on whether the ADM is prehydrated (19-23).

Preoperatively, the inframammary fold is marked with the patient in an upright position, as is the perimeter of the breast dimensions. If the inframammary fold has been violated during the mastectomy, it is marked on the inside of the mastectomy flap using methylene blue. The inferior mastectomy flap is then compressed against the chest wall to imprint the marking and thus provide a guide for the level of fixation of the ADM to the chest wall. The dermal side of the ADM is oriented against the mastectomy flap. Interrupted 2-0 Vicryl threepoint sutures are used to affix the ADM from the inframammary fold to the chest wall along the inferior breast (Figure 4). The ADM is then curved laterally and superiorly along the lateral border of the breast perimeter to recreate the curvilinear origins of the breast mound. Here, sutures are placed from the ADM to the chest wall leaving the mastectomy flap free. 2-0 Vicryl sutures are used to join the inferior pectoralis muscle to the superior ADM medially and laterally, leaving a gap for insertion of an implant sizer. This opening is temporarily sutured closed and the skin stapled. The patient is then placed in a sitting position to determine adequacy of the position of the inframammary fold and shape of the breast mound. Once the ADM is inset and the breast shape and size are confirmed, the sizer is removed and running 2-0 PDS sutures are used to reinforce the fixation to the chest wall. Closed suction drains are placed laterally and inferiorly between 

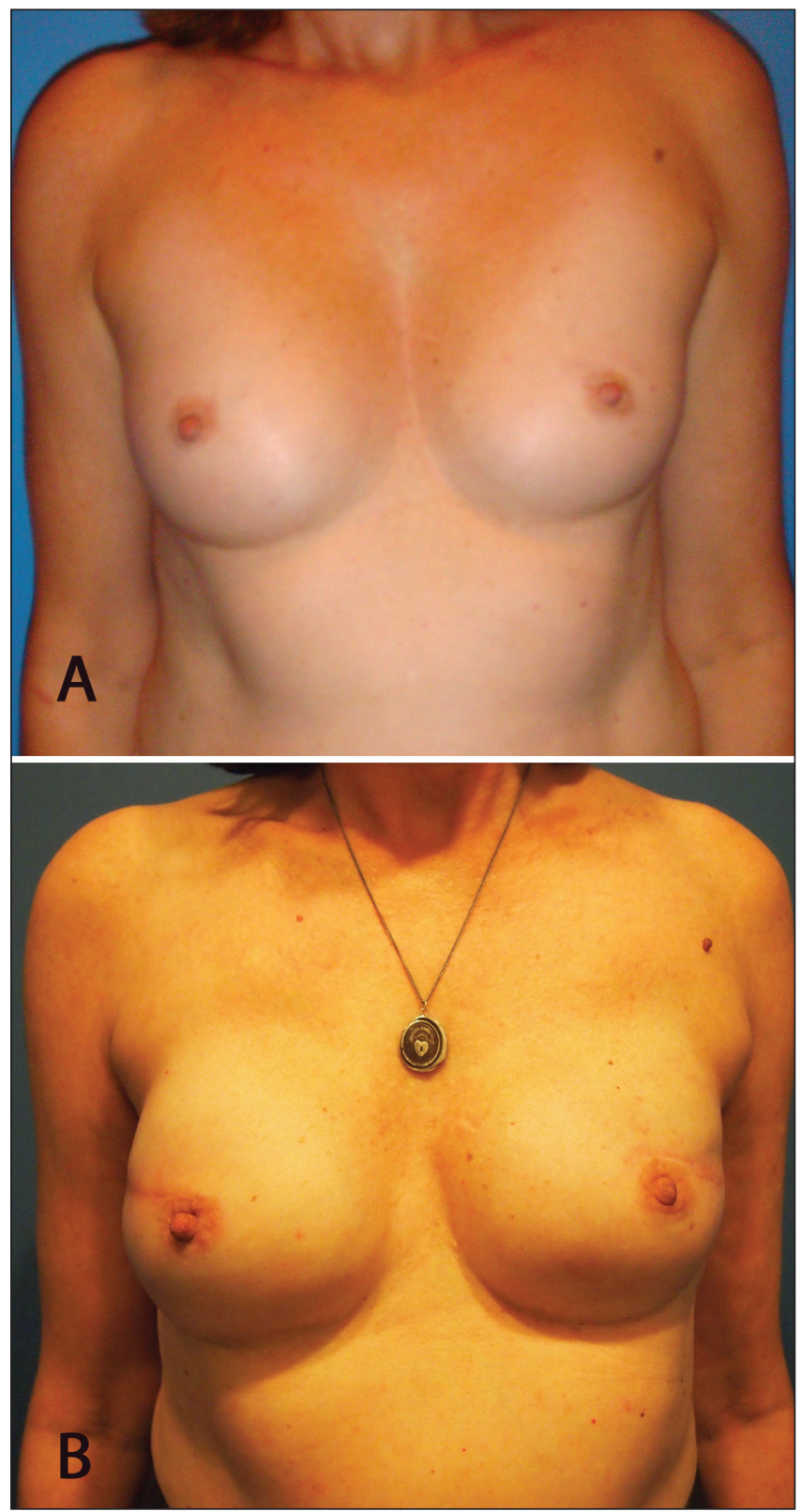

Figure 1) Bilateral direct-to-implant breast reconstruction. A A 60-year-old patient who underwent nipple-sparing direct-to-implant reconstruction using AlloDerm (LifeCell Corporation, USA) $6 \mathrm{~cm} \times 16 \mathrm{~cm}$ and round silicone gel implants (Style 15-304g Allergan [Allergan Inc, USA]). B Postoperative photograph

the ADM and the mastectomy flaps. The final implant is placed and closure is performed.

A summary of the publications reviewing outcomes after direct-toimplant breast reconstruction using ADMs is presented in Table 1 (11,24-31). Overall, direct-to-implant reconstruction outcomes compare favourably with the Mentor and Allergan Core Study Results, which track complications and revision rates following two-stage implant reconstruction without $\operatorname{ADM}(32,33)$. On short-term follow-up, weighted analysis of the studies in Table 1 shows direct-to-implant reconstruction to have lower rates of capsular contracture $(0.3 \%$ versus $8.3 \%$ to $17.1 \%)$, seroma $(1.2 \%$ versus $4.9 \%)$, infection $(1.4 \%$ versus $3.2 \%$ to $5.7 \%)$, late revision $(8.5 \%$ versus $27 \%$ to $53.3 \%)$ and implant loss $(1.5 \%$ versus $5.7 \%$ to $7.7 \%$ ) compared with the Core Studies. Higher rates of mastectomy flap necrosis $(4.7 \%$ versus $2.3 \%$ ) may be related to increased tension placed on the skin closure with immediate placement of the permanent prosthesis. Many authors have noted that

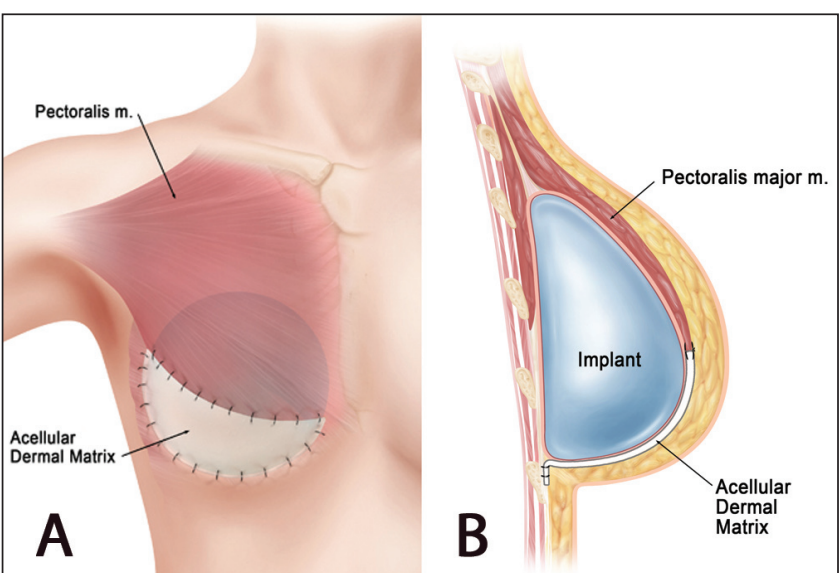

Figure 2) A Acellular dermal matrix coverage of implant (anteroposterior). B Acellular dermal matrix coverage of implant (lateral). $m$ Muscle

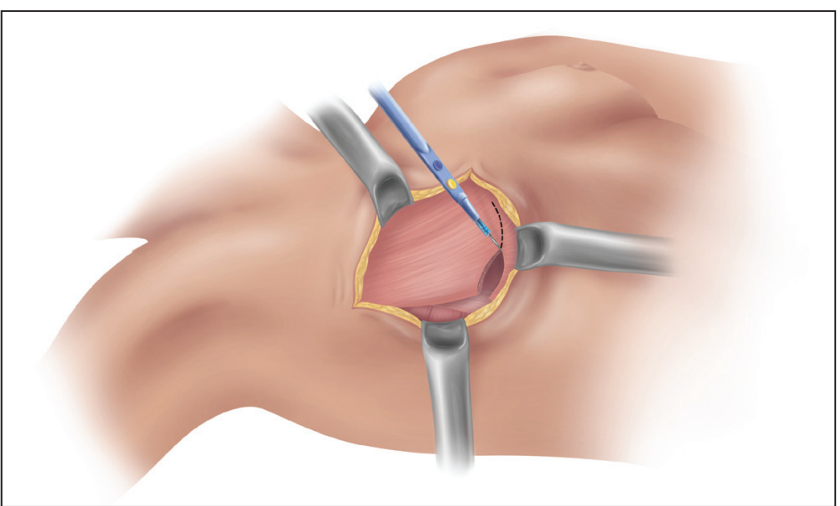

Figure 3) Elevation of the pectoralis muscle in preparation for placement of implant and acellular dermal matrix

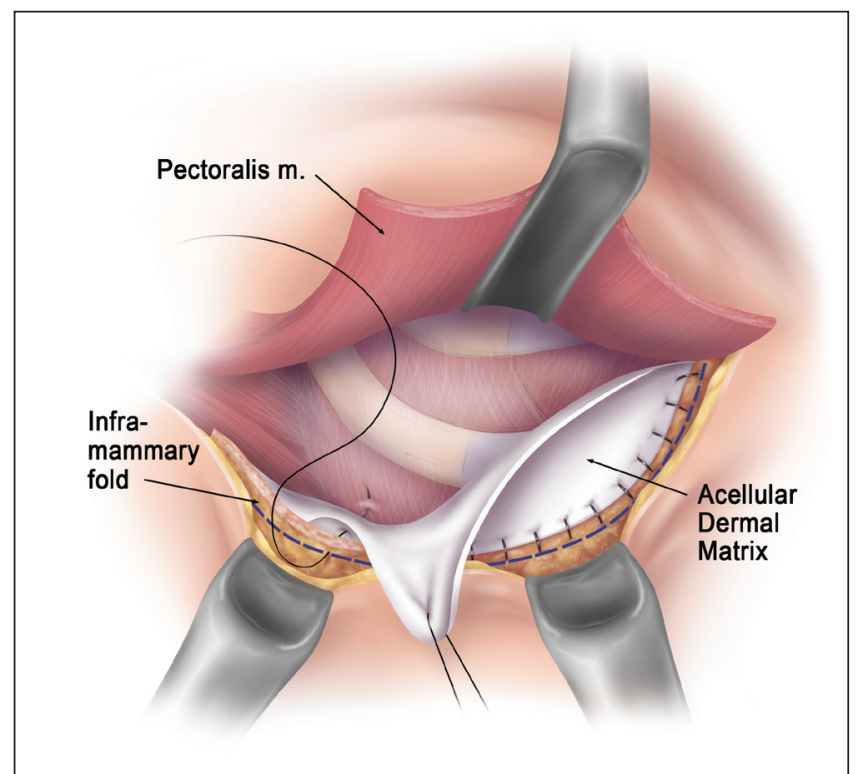

Figure 4) Fixation of acellular dermal matrix to chest wall and inframammary fold using three-point suture. $m$ Muscle

complications tend to occur more frequently earlier in their experience with this technique. Complication rates may be seen to decrease as an individual surgeon's experience increases. The ability to accurately detect the health of the mastectomy flaps at the time of implant placement remains a challenge and this may be aided in the future with perfusion imaging devices $(26,34)$. 
TABLE 1

\begin{tabular}{|c|c|c|c|c|c|c|c|c|c|c|}
\hline $\begin{array}{l}\text { Author (reference), } \\
\text { country }\end{array}$ & Title & $\begin{array}{c}\text { Acellular } \\
\text { dermal } \\
\text { matrix }\end{array}$ & Sample & Follow-up & $\begin{array}{l}\text { Mastectomy } \\
\text { flap necrosis } \\
\text { requiring } \\
\text { revision, \% }\end{array}$ & $\begin{array}{l}\text { Capsular } \\
\text { contracture } \\
\text { rate, } \%\end{array}$ & $\begin{array}{l}\text { Late revision } \\
\text { rate, } \%\end{array}$ & $\begin{array}{l}\text { Seroma } \\
\text { rate, \% }\end{array}$ & $\begin{array}{c}\text { Infection } \\
\text { rate, } \%\end{array}$ & $\begin{array}{c}\text { Implant } \\
\text { extrusion } \\
\text { or require- } \\
\text { ment for } \\
\text { autologous } \\
\text { salvage, \% }\end{array}$ \\
\hline $\begin{array}{l}\text { Ashikari et al (24), } \\
\text { USA }\end{array}$ & $\begin{array}{l}\text { Subcutaneous } \\
\text { mastectomy and } \\
\text { immediate } \\
\text { reconstruction for } \\
\text { prevention of breast } \\
\text { cancer for high-risk } \\
\text { patients }\end{array}$ & $\begin{array}{c}\text { AlloDerm }^{\circledR *} \\
4 \mathrm{~cm} \times \\
12 \mathrm{~cm}\end{array}$ & $\begin{array}{c}65 \text { patients, } \\
130 \text { breasts } \\
\text { One stage }\end{array}$ & $\begin{array}{c}4.6 \text { years } \pm \\
56 \text { months }\end{array}$ & N/D & N/D & $\mathrm{N} / \mathrm{D}$ & N/D & 0 & $\mathrm{~N} / \mathrm{D}$ \\
\hline $\begin{array}{l}\text { Austen et al (25), } \\
\text { USA }\end{array}$ & $\begin{array}{l}\text { A simplified technique } \\
\text { for single stage } \\
\text { breast } \\
\text { reconstruction }\end{array}$ & $\begin{array}{c}\text { AlloDerm }{ }^{\circledR} \\
4 \mathrm{~cm} \times \\
12 \mathrm{~cm} \\
4 \mathrm{~cm} \times \\
16 \mathrm{~cm}\end{array}$ & $\begin{array}{l}25 \text { patients, } \\
35 \text { breasts } \\
\text { One stage }\end{array}$ & 10 months & $\begin{array}{c}5.7 \\
(2 \text { cases } \\
\text { revised in } \\
\text { office setting })\end{array}$ & N/D & $\begin{array}{l}8.6 \\
(1 \text { implant } \\
\text { exchanged } \\
\text { for asymme- } \\
\text { try, one for } \\
\text { rippling) }\end{array}$ & N/D & N/D & 2.9 \\
\hline $\begin{array}{l}\text { Breuing et al (11), } \\
\text { USA }\end{array}$ & $\begin{array}{l}\text { Immediate bilateral } \\
\text { breast } \\
\text { reconstruction with } \\
\text { implants and } \\
\text { inferolateral } \\
\text { AlloDerm slings }\end{array}$ & $\begin{array}{l}\text { AlloDerm }^{\circledR} \\
4-6 \mathrm{~cm} \times \\
14-16 \mathrm{~cm}\end{array}$ & $\begin{array}{l}10 \text { patients, } \\
20 \text { breasts } \\
\text { One stage }\end{array}$ & $\begin{array}{l}6 \text { months } \\
\text { to } 1 \text { year }\end{array}$ & $\begin{array}{c}5 \\
\text { (Revised in } \\
\text { office setting) }\end{array}$ & 0 & 0 & 0 & 0 & 0 \\
\hline $\begin{array}{l}\text { Colwell et al (26), } \\
\text { USA }\end{array}$ & $\begin{array}{l}\text { Retrospective review } \\
\text { of } 331 \text { consecutive } \\
\text { immediate single } \\
\text { stage implant } \\
\text { reconstructions with } \\
\text { acellular dermal } \\
\text { matrix: Indications, } \\
\text { complications, } \\
\text { trends and costs }\end{array}$ & AlloDerm $^{\circledR}$ & $\begin{array}{l}211 \text { patients, } \\
331 \text { breasts } \\
\text { One stage }\end{array}$ & $N / D$ & 9.1 & N/D & $\mathrm{N} / \mathrm{D}$ & 1.5 & 3.0 & 1.5 \\
\hline $\begin{array}{l}\text { Gamboa-Bobadilla } \\
\text { (27), USA }\end{array}$ & $\begin{array}{l}\text { Implant breast } \\
\text { reconstruction using } \\
\text { acellular dermal } \\
\text { matrix }\end{array}$ & $\begin{array}{c}\text { AlloDerm }^{\circledR} \\
4 \mathrm{~cm} \times \\
16 \mathrm{~cm}\end{array}$ & $\begin{array}{l}11 \text { patients, } \\
13 \text { breasts } \\
\text { One stage }\end{array}$ & 14 months & N/D & $N / D$ & $\mathrm{~N} / \mathrm{D}$ & 7.7 & 7.7 & 7.7 \\
\hline $\begin{array}{l}\text { Salzberg et al (28), } \\
\text { USA }\end{array}$ & $\begin{array}{l}\text { An 8-year experience } \\
\text { of direct-to-implant } \\
\text { immediate breast } \\
\text { reconstruction using } \\
\text { human acellular } \\
\text { dermal matrix } \\
\text { (AlloDerm) }\end{array}$ & AlloDerm ${ }^{\circledR}$ & $\begin{array}{c}260 \text { patients, } \\
466 \text { breasts } \\
\text { One stage }\end{array}$ & $\begin{array}{c}28.9 \pm 21.3 \\
\text { months }\end{array}$ & 1.1 & $\begin{array}{c}0.4 \\
\text { Both cases } \\
\quad(2) \\
\text { required } \\
\text { operative } \\
\text { interven- } \\
\text { tion }\end{array}$ & $\begin{array}{c}9.4 \\
\text { (Most frequent } \\
\text { revision was } \\
\text { to increase } \\
\text { implant size) }\end{array}$ & N/D & 0.2 & 1.3 \\
\hline $\begin{array}{l}\text { Salzberg et al (29), } \\
\text { USA }\end{array}$ & $\begin{array}{l}\text { Nonexpansive } \\
\text { immediate breast } \\
\text { reconstruction using } \\
\text { human acellular } \\
\text { tissue matrix graft } \\
\text { (AlloDerm) }\end{array}$ & AlloDerm ${ }^{\circledR}$ & $\begin{array}{l}49 \text { patients, } \\
76 \text { breasts } \\
\text { One stage }\end{array}$ & $\begin{array}{c}18 \text { months } \\
\text { (range } \\
3-52 \\
\text { months) }\end{array}$ & $\begin{array}{c}3.9 \\
\text { (2 managed } \\
\text { conservatively } \\
\text { with dressings, } \\
\text { one managed } \\
\text { operatively) }\end{array}$ & 0 & $\mathrm{~N} / \mathrm{D}$ & 0 & 0 & 0 \\
\hline $\begin{array}{l}\text { Topol et al (30), } \\
\text { USA }\end{array}$ & $\begin{array}{l}\text { Immediate single- } \\
\text { stage breast } \\
\text { reconstruction using } \\
\text { implants and human } \\
\text { acellular dermal } \\
\text { tissue matrix with } \\
\text { adjustment of the } \\
\text { lower pole of the } \\
\text { breast to reduce } \\
\text { unwanted lift }\end{array}$ & $\begin{array}{c}\text { AlloDerm } \\
4 \mathrm{~cm} \times \\
16 \mathrm{~cm}\end{array}$ & $\begin{array}{l}23 \text { patients, } \\
35 \text { breasts } \\
\text { One stage }\end{array}$ & $\begin{array}{l}9.5 \text { months } \\
\text { (range } \\
1-24 \\
\text { months) }\end{array}$ & 0 & 0 & $\mathrm{~N} / \mathrm{D}$ & 0 & 8.6 & 5.7 \\
\hline
\end{tabular}




\begin{tabular}{|c|c|c|c|c|c|c|c|c|c|c|}
\hline $\begin{array}{l}\text { Author (reference), } \\
\text { year }\end{array}$ & Title & $\begin{array}{c}\text { Acellular } \\
\text { dermal } \\
\text { matrix }\end{array}$ & Sample & Follow-up & $\begin{array}{l}\text { Mastectomy } \\
\text { flap necrosis } \\
\text { requiring } \\
\text { revision, } \% \\
\end{array}$ & $\begin{array}{c}\text { Capsular } \\
\text { contracture } \\
\text { rate, \% } \\
\end{array}$ & $\begin{array}{l}\text { Late revision } \\
\text { rate, } \%\end{array}$ & $\begin{array}{l}\text { Seroma } \\
\text { rate, } \%\end{array}$ & $\begin{array}{c}\text { Infection } \\
\text { rate, } \%\end{array}$ & $\begin{array}{c}\text { Implant } \\
\text { extrusion } \\
\text { or require- } \\
\text { ment for } \\
\text { autologous } \\
\text { salvage, \% }\end{array}$ \\
\hline $\begin{array}{l}\text { Zienowicz and } \\
\text { Karacaoglu (31), } \\
\text { USA }\end{array}$ & $\begin{array}{l}\text { Implant-based breast } \\
\text { reconstruction with } \\
\text { allograft }\end{array}$ & AlloDerm $^{\circledR}$ & $\begin{array}{l}24 \text { patients, } \\
30 \text { breasts } \\
\text { One stage } \\
\text { with adjust- } \\
\text { able implant }\end{array}$ & $\begin{array}{l}18 \text { months } \\
\text { (range } \\
15-24 \\
\text { months) }\end{array}$ & $\begin{array}{c}20 \\
\text { (All managed } \\
\text { conservatively) }\end{array}$ & 0 & 0 & 0 & 0 & 0 \\
\hline 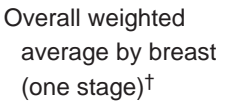 & & & & & $\begin{array}{c}4.7 \\
(47 / 993)\end{array}$ & $\begin{array}{c}0.3 \\
(2 / 627)\end{array}$ & $\begin{array}{c}8.5 \\
(47 / 551)\end{array}$ & $\begin{array}{c}1.2 \\
(6 / 505)\end{array}$ & $\begin{array}{c}1.4 \\
(15 / 1101)\end{array}$ & $\begin{array}{c}1.5 \\
(15 / 1006)\end{array}$ \\
\hline $\begin{array}{l}\text { Mentor }{ }^{\S} \text { Core Study } \\
\text { for Primary } \\
\text { Reconstruction } \\
\text { (25) } \\
\text { (3-year follow-up; } \\
\text { n=251) }\end{array}$ & & & & & N/D & 8.3 & 27 & 4.9 & 5.7 & 5.7 \\
\hline
\end{tabular}

Note: weighted averages calculated by breast. ${ }^{*}$ LifeCell Corporation, USA; ${ }^{\dagger}$ Studies with a mix of one- and two-stage reconstructions, or studies that did not report results by breast not included in weighted average; ${ }^{\ddagger}$ Allergan Inc, USA; ${ }^{\S}$ Mentor Worldwide LLC, USA. N/D Not documented

\section{Use in two-stage implant reconstruction}

The incorporation of an ADM into the initial stage of a two-stage alloplastic reconstruction has several purported benefits. Release of the pectoral origin and placement of ADM over the inferior surface of the tissue expander allows for more rapid tissue expansion, eliminates elevation of the serratus anterior for inferolateral coverage and may decrease the pain of the expansion phase. Many surgeons believe that incorporation of ADMs at the tissue expander stage enables more precise placement of the tissue expander and allows for better inferior pole expansion and projection. The majority of surgeons, therefore, continue to perform two-stage reconstruction with ADMs as an adjunct in an attempt to improve aesthetic outcomes.

The ideal patient characteristics are similar to those outlined for direct-to-implant reconstruction; however, patients with larger and more ptotic breasts may be included in this category. At our institution, patients undergoing postoperative radiation therapy are also candidates (Figures 5A and 5B). Our rationale for this is that patients undergoing radiation therapy (which takes place after inflation of the tissue expander) may experience early tightening of the capsule. Provision of a second stage allows for modifications before placement of the permanent prosthesis.

The surgical technique used for two-stage reconstruction using ADMs is identical to that outlined for direct-to-implant reconstruction; however, after verification of shape and position of the inframammary fold with a temporary sizer, an appropriately sized tissue expander is placed in the subpectoral/sub-ADM pocket (Figure 6). Enough saline is infiltrated to compress the ADM to the mastectomy flaps without compromising tissue viability. Tissue expansion commences one week postoperatively in the absence of mastectomy flap necrosis or wound dehiscence. Implant exchange is performed three months following complete expansion or three months following termination of radiation. We have seen wound dehiscence when the original incision is used for the second stage access incision in the setting of radiation. We now routinely use an incision remote from the mastectomy incision (usually in the inframammary fold) for placement of the final prosthesis in a patient who has undergone radiation after tissue expander/ADM insertion and expansion.

Table 2 summarizes studies that have documented outcomes after immediate two-stage alloplastic reconstruction using ADMs (12,3548). Overall, weighted analysis shows higher rates of seroma $(5.8 \%$ versus $4.9 \%$ ), infection ( $5.3 \%$ versus $3.2 \%$ to $5.7 \%$ ) and mastectomy flap necrosis $(7.6 \%$ versus $2.3 \%)$ when comparing two-stage reconstruction with ADM with the Core Studies (ie, two-stage reconstruction without ADM). There is a comparable rate of implant extrusion ( $4.9 \%$ versus $5.7 \%$ to $7.7 \%$ ), and lower rates of capsular contracture ( $2.6 \%$ versus $8.3 \%$ to $17.1 \%$ ) and late revision ( $10.7 \%$ versus $27 \%$ to $53 \%$ ). In 2011 , a meta-analysis performed by Kim et al (49) compared 19 studies reporting two-stage reconstruction using ADM $(\mathrm{n}=2037)$ with 35 studies reporting traditional non-ADM twostage reconstruction $(n=12,847)$. These authors found higher rates of seroma $(4.8 \%$ versus $3.5 \%)$, infection $(5.3 \%$ versus $4.7 \%$ ) and mastectomy flap necrosis $(6.9 \%$ versus $4.9 \%)$ in the ADM patient group. There was an equivalent rate of reconstructive failure $(3.8 \%)$. These numbers correspond closely with those from our weighted analysis (Table 2). Sbitany and Serletti (50) reviewed six articles comparing outcomes of two-stage expander/implant reconstruction using ADM versus two-stage expander/implant reconstruction without ADM. These authors found a higher rate of seroma in the ADM group (8.4\% ADM verus $4.3 \%$ non-ADM), infection $(3.4 \%$ ADM versus $2.8 \%$ non-ADM [P not significant]) and mastectomy flap necrosis (9.3\% ADM versus $7.2 \%$ non-ADM [P not significant]) and similar rates of reconstructive failure (3.4\% ADM, 3.2\% nonADM). Hoppe et al (51) performed another meta-analysis published in 2011. These authors reviewed eight studies comparing outcomes of two-stage expander/implant reconstruction using ADM versus two-stage expander/implant reconstruction without ADM and found increased odds of seroma, hematoma, infection and explantation in the ADM group.

Given the heterogeneity of studies included in these reviews, it is difficult to draw definitive conclusions regarding outcomes. Two-stage 
TABLE 2

\begin{tabular}{|c|c|c|c|c|c|c|c|c|c|c|}
\hline $\begin{array}{l}\text { Author } \\
\text { (reference), } \\
\text { country }\end{array}$ & Title & $\begin{array}{l}\text { Acellular } \\
\text { dermal } \\
\text { matrix }\end{array}$ & Sample & Follow-up & $\begin{array}{l}\text { Mastectomy } \\
\text { flap necrosis } \\
\text { requiring } \\
\text { revision, \% }\end{array}$ & $\begin{array}{c}\text { Capsular } \\
\text { contracture } \\
\text { rate, } \%\end{array}$ & $\begin{array}{l}\text { Late } \\
\text { revision } \\
\text { rate, } \%\end{array}$ & $\begin{array}{l}\text { Seroma } \\
\text { rate, } \%\end{array}$ & $\begin{array}{l}\text { Infection } \\
\text { rate, } \%\end{array}$ & $\begin{array}{c}\text { Implant } \\
\text { extrusion or } \\
\text { requirement } \\
\text { for } \\
\text { autologous } \\
\text { salvage, \% }\end{array}$ \\
\hline $\begin{array}{c}\text { Antony et al } \\
(35), \text { USA }\end{array}$ & $\begin{array}{l}\text { Acellular human der- } \\
\text { mis implantation in } \\
153 \text { immediate } \\
\text { two-stage tissue } \\
\text { expander breast } \\
\text { reconstructions: } \\
\text { Determining the } \\
\text { incidence and sig- } \\
\text { nificant predictors } \\
\text { of complications }\end{array}$ & $\begin{array}{c}\text { AlloDerm }{ }^{\circledR *} \\
4 \mathrm{~cm} \times 16 \mathrm{~cm}\end{array}$ & $\begin{array}{c}96 \text { patients, } \\
153 \text { breasts } \\
\text { Two stage }\end{array}$ & $N / D$ & 4.6 & $N / D$ & $N / D$ & 7.2 & 3.3 & 5.9 \\
\hline $\begin{array}{c}\text { Becker et al } \\
(36), \text { USA }\end{array}$ & $\begin{array}{l}\text { AlloDerm versus } \\
\text { DermaMatrix in } \\
\text { immediate } \\
\text { expander-based } \\
\text { breast } \\
\text { reconstruction: } \\
\text { A preliminary } \\
\text { comparison of } \\
\text { complication } \\
\text { profiles and } \\
\text { material } \\
\text { compliance }\end{array}$ & $\begin{array}{c}\text { AlloDerm }^{\circledR} \\
25 \text { breasts } \\
\text { DermaMatrix }^{\dagger} \\
25 \text { breasts }\end{array}$ & $\begin{array}{l}30 \text { patients, } \\
50 \text { breasts } \\
\text { Two stage }\end{array}$ & 6.7 months & 0 & $N / D$ & $N / D$ & $\begin{array}{c}4 \\
\text { (1 breast in } \\
\text { DermaMatrix } \\
\text { group) }\end{array}$ & $\begin{array}{c}4 \\
\text { (1 breast in } \\
\text { DermaMatrix } \\
\text { group) }\end{array}$ & 0 \\
\hline $\begin{array}{l}\text { Bindingnavele } \\
\text { et al (12), USA }\end{array}$ & $\begin{array}{l}\text { Use of acellular } \\
\text { cadaveric dermis } \\
\text { and tissue } \\
\text { expansion in } \\
\text { postmastectomy } \\
\text { breast } \\
\text { reconstruction }\end{array}$ & AlloDerm ${ }^{\circledR}$ & $\begin{array}{l}41 \text { patients, } \\
65 \text { breasts } \\
\text { Two stage }\end{array}$ & $\begin{array}{c}10 \text { months } \\
\text { (range } \\
7-21 \\
\text { months) }\end{array}$ & 0 & 0 & $N / D$ & 4.6 & 3.1 & 1.5 \\
\hline $\begin{array}{l}\text { Hanna et al (37), } \\
\text { USA (reported } \\
\text { by patient) }\end{array}$ & $\begin{array}{l}\text { Comparison of two } \\
\text { types of expander- } \\
\text { based } \\
\text { reconstruction: } \\
\text { Acellular dermal } \\
\text { matrix-assisted vs } \\
\text { total submuscular } \\
\text { placement }\end{array}$ & $\begin{array}{c}\text { AlloDerm }{ }^{\circledR} \\
4 \mathrm{~cm} \times \\
16 \mathrm{~cm}\end{array}$ & $\begin{array}{l}31 \text { patients } \\
\text { Two stage }\end{array}$ & 7.7 months & 6.5 & $N / D$ & $N / D$ & 19.4 & 16.1 & 16.1 \\
\hline $\begin{array}{l}\text { Lanier et al } \\
(38), \text { USA }\end{array}$ & $\begin{array}{l}\text { The effect of } \\
\text { acellular dermal } \\
\text { matrix use on } \\
\text { complication rates } \\
\text { in tissue } \\
\text { expander/implant } \\
\text { breast } \\
\text { reconstruction }\end{array}$ & $\begin{array}{c}\text { AlloDerm }{ }^{\circledR} \\
\text { Strattice* } \\
\text { FlexHD } \\
6 \mathrm{~cm} \times 16 \mathrm{~cm} \\
8 \mathrm{~cm} \times 16 \mathrm{~cm}\end{array}$ & $\begin{array}{l}52 \text { breasts } \\
\text { Two stage }\end{array}$ & $\begin{array}{l}6.8 \pm 2.8 \\
\text { months }\end{array}$ & 15.4 & 3.9 & 25 & 15.4 & 28.9 & 19.2 \\
\hline $\begin{array}{l}\text { Liu et al (39), } \\
\text { USA }\end{array}$ & $\begin{array}{l}\text { Postoperative } \\
\text { complications in } \\
\text { prosthesis-based } \\
\text { breast } \\
\text { reconstruction } \\
\text { using acellular } \\
\text { dermal matrix }\end{array}$ & AlloDerm $^{\circledR} 1$ & $\begin{array}{c}192 \text { patients, } \\
266 \text { breasts } \\
\text { Two stage }\end{array}$ & $\mathrm{N} / \mathrm{D}$ & 13.9 & $N / D$ & $N / D$ & 7.1 & 6.8 & 4.9 \\
\hline $\begin{array}{l}\text { Losken (40), } \\
\text { USA }\end{array}$ & $\begin{array}{l}\text { Early results using } \\
\text { sterilized acellular } \\
\text { human dermis } \\
\text { (NeoForm) in post- } \\
\text { mastectomy tissue } \\
\text { expander breast } \\
\text { reconstruction }\end{array}$ & $\begin{array}{c}\text { SurgiMend } \\
\text { (previously } \\
\text { Neoform) } \\
4 \mathrm{~cm} \times 16 \mathrm{~cm} \\
6 \mathrm{~cm} \times 16 \mathrm{~cm}\end{array}$ & $\begin{array}{l}22 \text { patients, } \\
31 \text { breasts } \\
\text { Two stage }\end{array}$ & $\begin{array}{c}10.2 \\
\text { months } \\
\text { (range } \\
6-16 \\
\text { months) }\end{array}$ & $\begin{array}{c}3.2 \\
\text { (Managed in } \\
\text { office setting) }\end{array}$ & $\mathrm{N} / \mathrm{D}$ & $\mathrm{N} / \mathrm{D}$ & 0 & 0 & 0 \\
\hline $\begin{array}{c}\text { Margulies et al } \\
\text { (41), USA }\end{array}$ & $\begin{array}{l}\text { Total skin-sparing } \\
\text { mastectomy with- } \\
\text { out preservation of } \\
\text { the nipple-areola } \\
\text { complex }\end{array}$ & AlloDerm $^{\circledR}$ & $\begin{array}{l}31 \text { patients, } \\
50 \text { breasts } \\
\text { Two stage }\end{array}$ & $\begin{array}{l}7.9 \pm 5.4 \\
\text { months }\end{array}$ & 14 & $N / D$ & $N / D$ & $N / D$ & 4 & $N / D$ \\
\hline
\end{tabular}




\begin{tabular}{|c|c|c|c|c|c|c|c|c|c|c|}
\hline $\begin{array}{l}\text { Author } \\
\text { (reference), } \\
\text { country }\end{array}$ & Title & $\begin{array}{c}\text { Acellular } \\
\text { dermal } \\
\text { matrix }\end{array}$ & Sample & Follow-up & $\begin{array}{l}\text { Mastectomy } \\
\text { flap necrosis } \\
\text { requiring } \\
\text { revision, } \%\end{array}$ & $\begin{array}{l}\text { Capsular } \\
\text { contracture } \\
\text { rate, } \%\end{array}$ & $\begin{array}{l}\text { Late } \\
\text { revision } \\
\text { rate, \% }\end{array}$ & $\begin{array}{l}\text { Seroma } \\
\text { rate, \% }\end{array}$ & $\begin{array}{c}\text { Infection } \\
\text { rate, \% }\end{array}$ & $\begin{array}{c}\text { Implant } \\
\text { extrusion or } \\
\text { requirement } \\
\text { for } \\
\text { autologous } \\
\text { salvage, \% }\end{array}$ \\
\hline $\begin{array}{l}\text { Nahabedian } \\
\text { et al (42), } \\
\text { USA }\end{array}$ & $\begin{array}{l}\text { AlloDerm perfor- } \\
\text { mance in the set- } \\
\text { ting of prosthetic } \\
\text { breast surgery, } \\
\text { infection, and irra- } \\
\text { diation }\end{array}$ & AlloDerm $^{\circledR}$ & $\begin{array}{c}76 \text { patients, } \\
100 \text { breasts } \\
\text { Two stage }\end{array}$ & $\begin{array}{l}17 \text { months } \\
\text { (range } \\
6-37 \\
\text { months) }\end{array}$ & 3 & $\mathrm{~N} / \mathrm{D}$ & N/D & 5 & 5 & 2 \\
\hline $\begin{array}{l}\text { Namnoum (43), } \\
\text { USA }\end{array}$ & $\begin{array}{l}\text { Expander/implant } \\
\text { reconstruction with } \\
\text { AlloDerm: Recent } \\
\text { experience }\end{array}$ & $\begin{array}{c}\text { AlloDerm }^{\circledR} \\
4 \mathrm{~cm} \times 16 \mathrm{~cm} \\
4 \mathrm{~cm} \times 12 \mathrm{~cm}\end{array}$ & $\begin{array}{c}20 \text { patients, } \\
29 \text { breasts } \\
\text { Two stage }\end{array}$ & $\begin{array}{l}21 \text { months } \\
\text { (range 3-32 } \\
\text { months) }\end{array}$ & 3.4 & 0 & 0 & 3.4 & 3.4 & 0 \\
\hline $\begin{array}{l}\text { Parikh (44), } \\
\text { USA }\end{array}$ & $\begin{array}{l}\text { Immediate breast } \\
\text { reconstruction with } \\
\text { tissue expanders } \\
\text { and alloderm }\end{array}$ & AlloDerm $^{\circledR}$ & $\begin{array}{l}43 \text { patients, } \\
58 \text { breasts } \\
\text { Two stage }\end{array}$ & N/D & 5.2 & $N / D$ & $N / D$ & 3.4 & 5.2 & 1.7 \\
\hline $\begin{array}{l}\text { Preminger et al } \\
(45), \text { USA }\end{array}$ & $\begin{array}{l}\text { The influence of } \\
\text { AlloDerm on } \\
\text { expander dynam- } \\
\text { ics and complica- } \\
\text { tions in the setting } \\
\text { of immediate tis- } \\
\text { sue expander/ } \\
\text { implant recon- } \\
\text { struction: A } \\
\text { matched-cohort } \\
\text { study }\end{array}$ & AlloDerm ${ }^{\circledR}$ & $\begin{array}{l}45 \text { breasts } \\
\text { Two stage }\end{array}$ & N/D & $N / D$ & $N / D$ & $N / D$ & 6.7 & 2.2 & 0 \\
\hline $\begin{array}{l}\text { Sbitany et al } \\
(46), \text { USA }\end{array}$ & $\begin{array}{l}\text { Acellular dermis- } \\
\text { assisted prosthetic } \\
\text { breast reconstruc- } \\
\text { tion versus com- } \\
\text { plete submuscular } \\
\text { coverage: A head- } \\
\text { to-head compari- } \\
\text { son of outcomes }\end{array}$ & AlloDerm ${ }^{\circledR}$ & $\begin{array}{l}50 \text { patients } \\
92 \text { breasts } \\
\text { Two stage }\end{array}$ & N/D & $N / D$ & $N / D$ & $N / D$ & 6 & 8 & 8 \\
\hline $\begin{array}{l}\text { Spear et al } \\
(47), \text { USA }\end{array}$ & $\begin{array}{l}\text { Acellular dermis- } \\
\text { assisted breast } \\
\text { reconstruction }\end{array}$ & $\begin{array}{c}\text { AlloDerm }{ }^{\circledR} \\
4 \mathrm{~cm} \times 12 \mathrm{~cm} \\
4 \mathrm{~cm} \times 16 \mathrm{~cm}\end{array}$ & $\begin{array}{l}43 \text { patients, } \\
58 \text { breasts } \\
\text { Two stage }\end{array}$ & $\begin{array}{c}25.9 \\
\text { months } \\
\text { (range } \\
19.2-35.3 \\
\text { months) }\end{array}$ & 3.4 & 2 & 4 & 1.7 & 6.9 & $\begin{array}{c}12.1 \\
\text { (Conversion to } \\
\text { autologous } \\
\text { salvage at } \\
\text { second stage } \\
\text { without implant } \\
\text { extrusion) }\end{array}$ \\
\hline $\begin{array}{l}\text { Vardanian et al } \\
\text { (48), USA }\end{array}$ & $\begin{array}{l}\text { Comparison of } \\
\text { implant based } \\
\text { immediate breast } \\
\text { reconstruction with } \\
\text { and without acellu- } \\
\text { lar dermal matrix }\end{array}$ & Alloderm $^{\circledR}$ & $\begin{array}{l}208 \text { breasts } \\
\text { Two stage }\end{array}$ & 29 months & N/D & 3.8 & N/D & N/D & 1 & N/D \\
\hline $\begin{array}{l}\text { Overall weighted } \\
\text { average by } \\
\text { breast (two } \\
\text { stage) }\end{array}$ & & & & & $\begin{array}{c}7.6 \\
(69 / 906)\end{array}$ & $\begin{array}{c}2.6 \\
(11 / 412)\end{array}$ & $\begin{array}{c}10.7 \\
(15 / 139)\end{array}$ & $\begin{array}{c}5.8 \\
(58 / 999)\end{array}$ & $\begin{array}{c}5.3 \\
(67 / 1257)\end{array}$ & $\begin{array}{c}4.9 \\
(50 / 1002)\end{array}$ \\
\hline $\begin{array}{l}\text { Allergan** Core } \\
\text { Study for } \\
\text { Primary } \\
\text { Reconstruction } \\
\text { (24) (7-year } \\
\text { follow-up; } n=98 \text { ) }\end{array}$ & & & & & 2.3 & 17.1 & 53.3 & N/D & 3.2 & 7.7 \\
\hline $\begin{array}{l}\text { Mentor }^{\dagger \dagger} \text { Core } \\
\text { Study for } \\
\text { Primary } \\
\text { Reconstruction } \\
\text { (25) (3-year } \\
\text { follow-up; } \\
n=251\end{array}$ & & & & & $N / D$ & 8.3 & 27 & 4.9 & 5.7 & 5.7 \\
\hline
\end{tabular}

Note: weighted averages calculated by breast. *LifeCell Corporation, USA; ${ }^{\dagger}$ Synthes Inc, USA; ${ }^{\ddagger}$ Ethicon, USA; ${ }^{\S} T E I$ Biosciences, USA; ${ }^{\top}$ Studies with a mix of oneand two-stage reconstructions and studies that did not report results by breast not included in weighted average; ${ }^{*}$ Allergan Inc, USA; ${ }^{+\dagger}$ Mentor Worldwide $L L C$, USA. N/D Not documented 

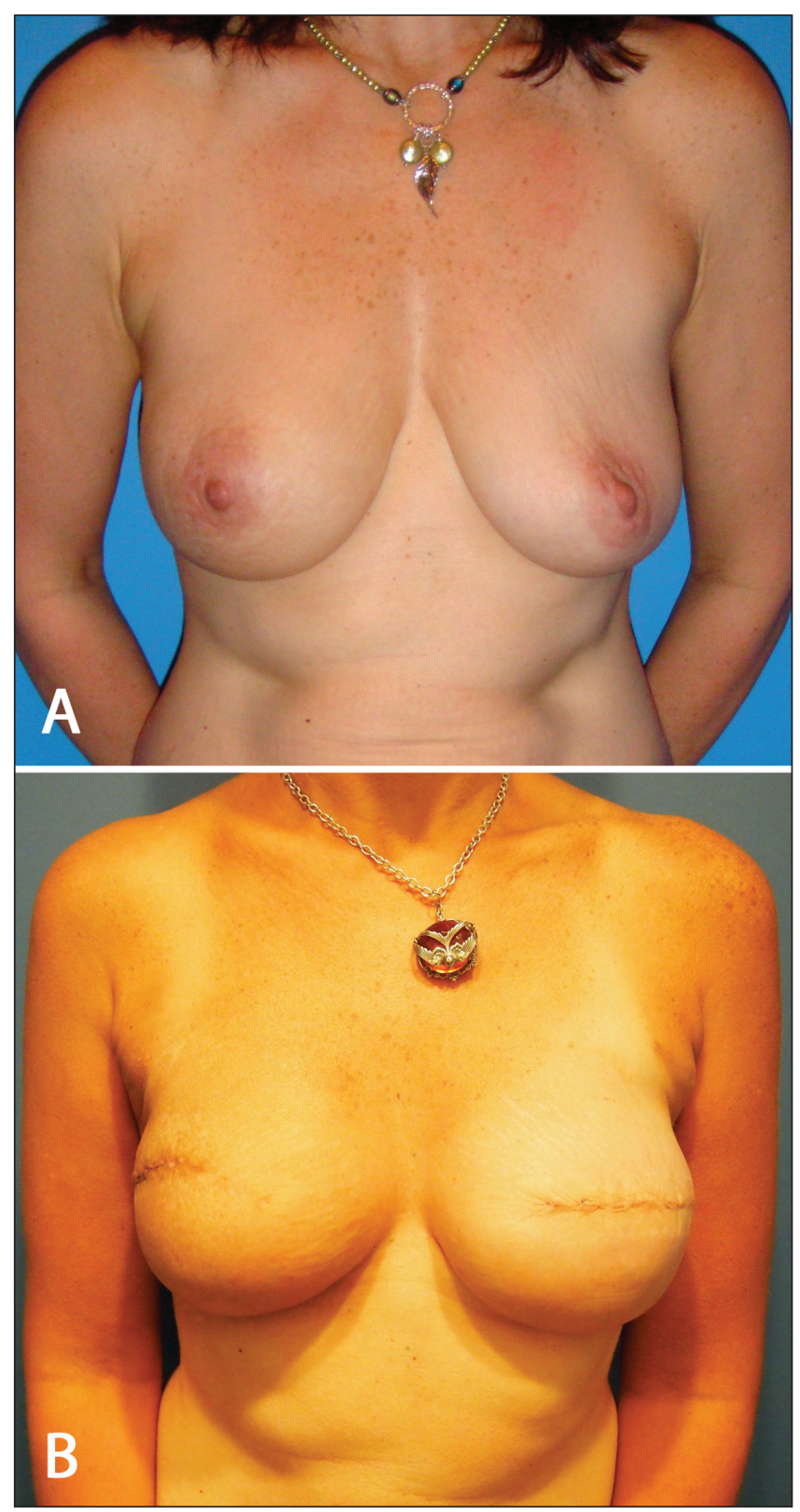

Figure 5) Breast reconstruction using acellular dermal matrices in the setting of chest wall irradiation. A A 46-year-old patient who underwent skinsparing mastectomies and tissue expander/AlloDerm (LifeCell Corporation, USA) reconstruction on the right and direct-to-implant reconstruction using AlloDerm and a shaped, cohesive silicone gel implant on the left (Style FX-410g Allergan [Allergan Inc, USA]). B Postoperative photograph taken one year after right-sided radiation and subsequent implant exchange for Style FX-450 (Allergan Inc, USA)

reconstruction with $\mathrm{ADMs}$ appears to be a safe alternative to traditional total submuscular coverage, and definitive comparisons can only be made using a randomized controlled trial format (McCarthy et al, Memorial Sloan Kettering Cancer Center [New York, USA], in progress).

\section{Use in correction of symmastia}

The Allergan Core Study reported a 41\% revision rate following a first revisionary surgery after breast augmentation (32). After primary aesthetic breast implant surgery, surgeons may be faced with thinning of tissues secondary to placement of large prostheses, surface irregularities, symmastia, implant displacement or 'bottoming out' and, most commonly, capsular contracture. Traditional revision techniques

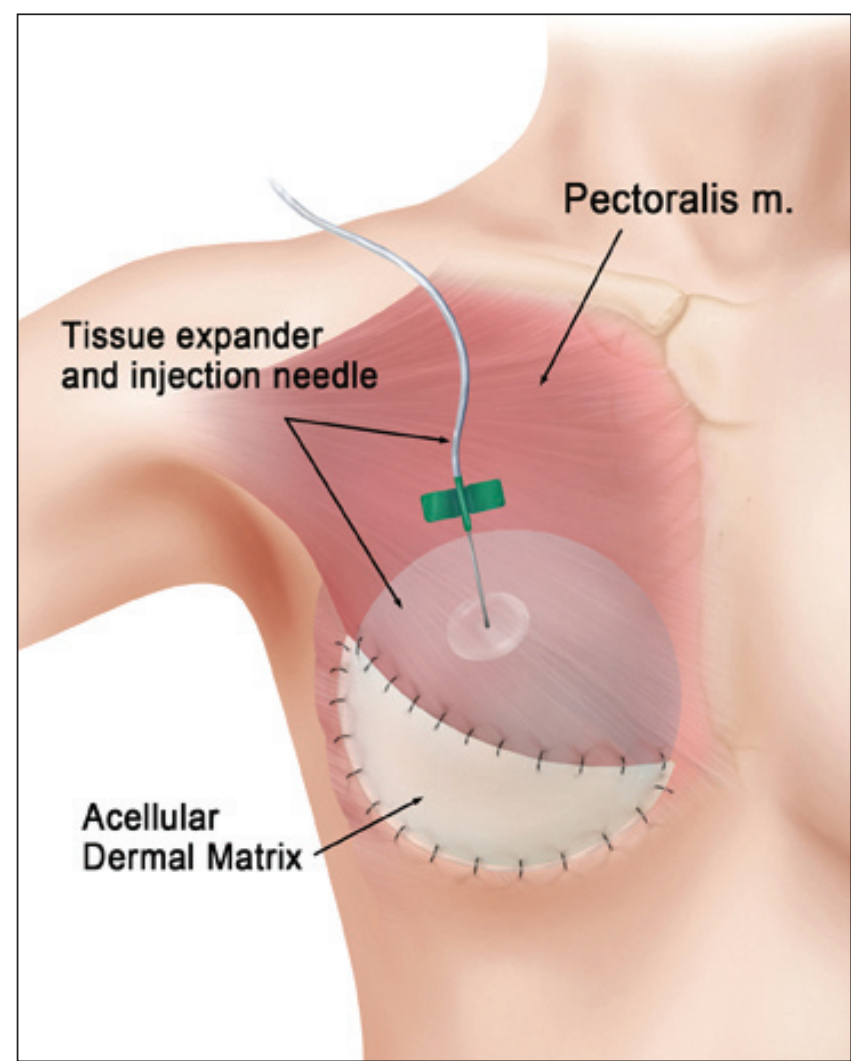

Figure 6) Acellular dermal matrix coverage of tissue expander (anteroposterior). $m$ Muscle



Figure 7) Medial placement of acellular dermal matrix to correct symmastia

include site change with creation of a neopocket, capsulorrhaphy, use of capsular flaps, fat grafting and conversion to form-stable prostheses $(52-55)$. 


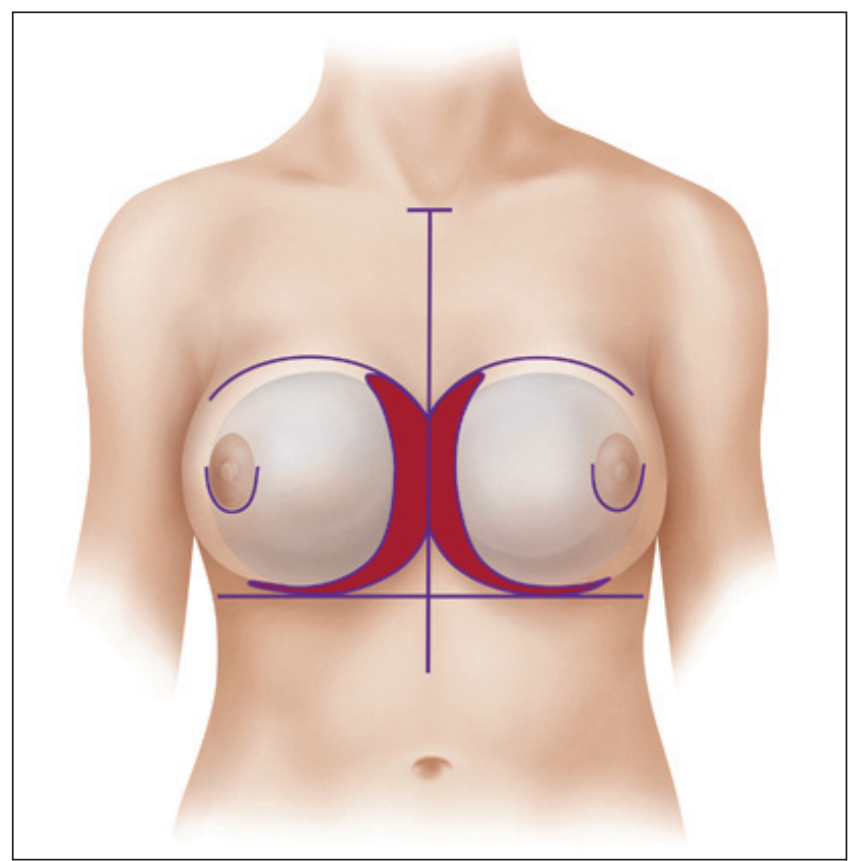

Figure 8) Preoperative markings in correction of symmastia (red-shaded areas correspond to medial implant malposition)

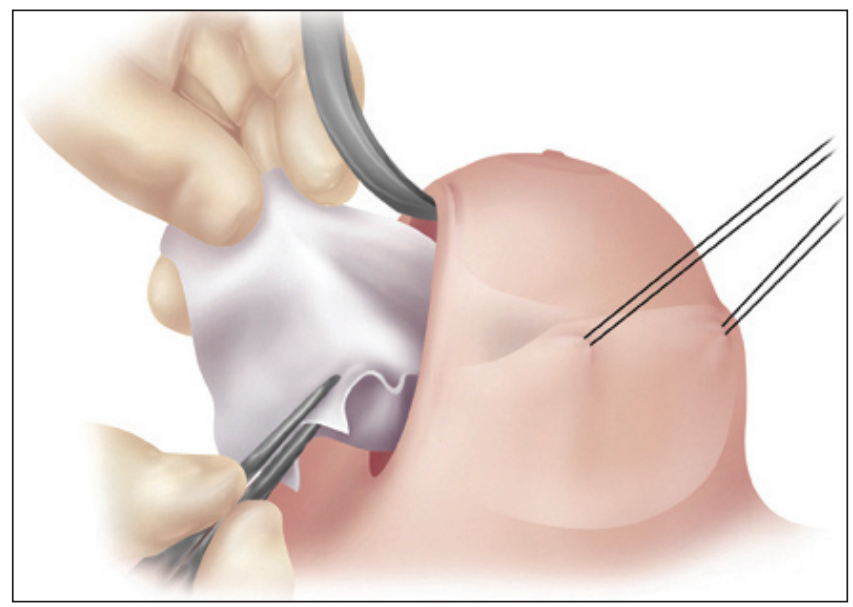

Figure 9) Intraoperative placement of acellular dermal matrix in correction of symmastia

Symmastia has traditionally been corrected by medial capsulorrhaphy with plication of the capsule to itself to bolster the implant laterally alone or site change with neopocket formation in addition to medial capsulorrhaphy. While these techniques have produced success in patients with tissue strong enough to support the correction with a new pocket and/or suturing alone, further revision surgery is common and many patients will have thin tissue leading to recurrence of the original problem. In these patients, use of an ADM to further buttress the implant into its new position can produce a long-lasting solution (Figure 7).

The technique includes preoperative marking of the desired medial border of the breast (Figure 8). In a patient with previous subpectoral implants, a site change to a neosubpectoral pocket is generally planned. The capsule is accessed via the pre-existing incision. A neo-subpectoral pocket is then dissected to the limits of the proposed new breast dimensions with the pre-existing implant in place. Once the new pocket has been created, the capsule is opened and the pre-existing implant is removed and irrigated in a triple antibiotic solution.

A medial capsulorrhaphy is performed to obliterate the previous pocket. The remaining anterior capsule is then plicated to the

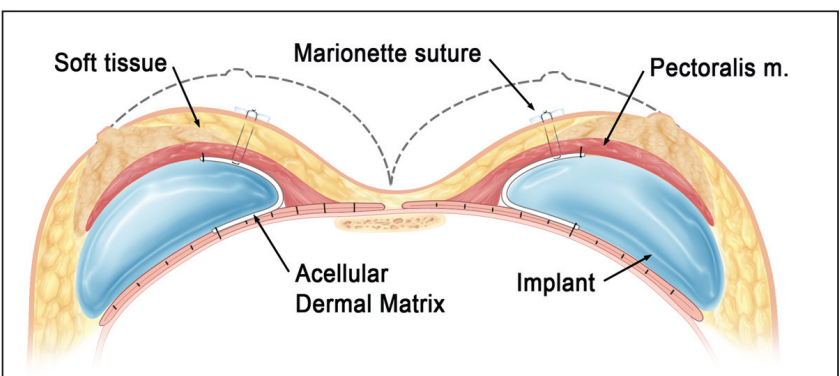

Figure 10) Placement of acellular dermal matrix to correct symmastia (dotted lines represent previous medial malposition). $m$ Muscle

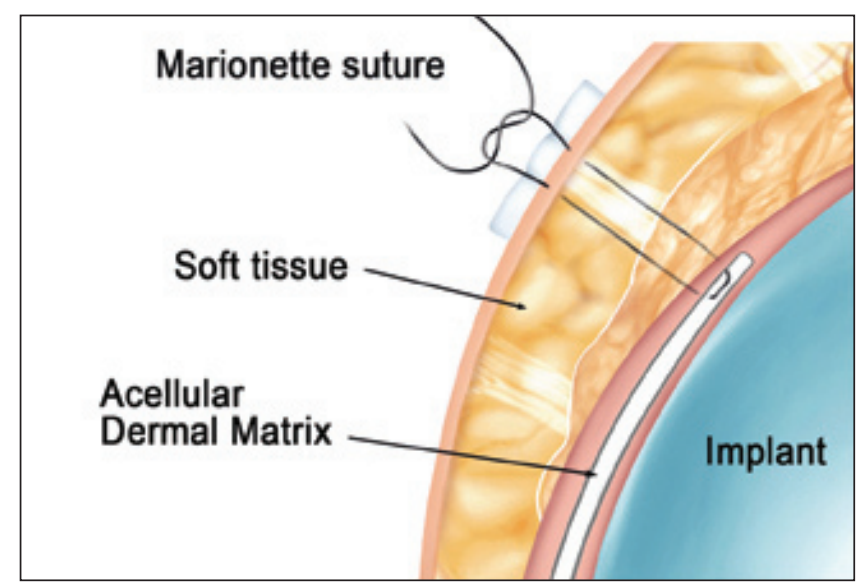

Figure 11) Placement of acellular dermal matrix to correct rippling

posterior capsule on the chest wall and the capsulotomy used to remove the implant is closed. Generally, a $4 \mathrm{~cm} \times 12 \mathrm{~cm}$ piece of ADM is used. A 2-0 prolene suture is placed from the upper corner of the $\mathrm{ADM}$ and brought into the neosubpectoral pocket and out through the breast skin using a Keith needle in a marionette fashion. A second marionette suture is placed identically to the lower corner of the ADM and brought out through the breast skin. The ADM is then 'parachuted' into the pocket using the marionette sutures, which are tied over bolsters (Jelonet [Smith \& Nephew, USA]) on the surface of the breast skin (Figure 9). The ADM is positioned anteriorly and medially abutting against the new, medial breast pocket and then draped around to the posterior chest wall and sutured in place. The posterior inferior corner of the ADM is sutured to the chest wall and interrupted sutures are used to suture the remaining edge of the ADM to the chest wall. A sizer is used to confirm that the ADM is properly aligned against the breast tissue with no gaps and that it controls the medial position of the implant (Figure 10). Sutures are used to affix the ADM to the anterior breast tissue. The final implant is placed after a drain is positioned between the breast tissue and the ADM. Bolster sutures are removed on postoperative day 7. Alternatively, the old pocket may be used with medial capsulorrhaphy and placement of the ADM for reinforcement.

Several authors have reported success in the use of ADM for correction of symmastia; however, large case series with long-term follow-up have not been performed (56-58).

\section{Use in correction of rippling}

Placement of large prostheses and thinning of periprosthetic tissue can lead to visible rippling and 'knuckling' on the skin surface in both reconstructive and aesthetic patients. In the past, techniques to camouflage skin surface irregularities have included fat grafting and conversion to form stable prostheses; however, in patients with extremely atrophic tissue, rippling may still be visible after these techniques are performed.

ADMs may be inserted in an overlay fashion between the implant and the capsule to augment the soft tissue and provide a barrier between the implant surface and the overlying breast tissue (Figure 11). This is 

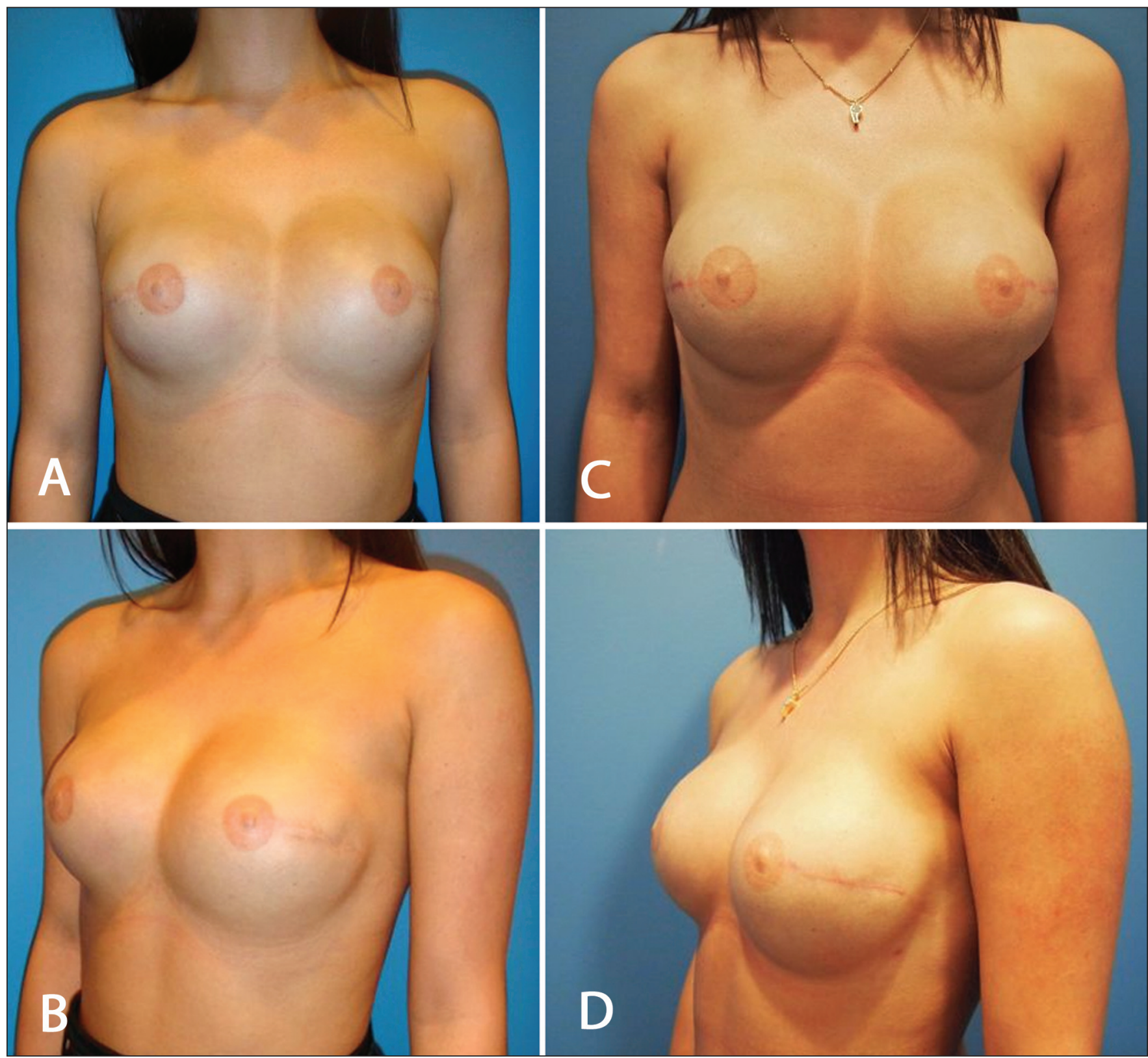

Figure 12) A 27-year-old patient who underwent bilateral mastectomies and immediate reconstruction using tissue expanders and subsequent textured round silicone gel implants $(500 \mathrm{~g})$. She experienced inferior displacement with blunting of the inframmamary fold and medial displacement of the left implant (A and B). She underwent conversion to smooth gel implants $(500 \mathrm{~g}$ ) and insertion of AlloDerm (LifeCell Corporation, USA) $6 \mathrm{~cm} \times 16 \mathrm{~cm}$ bilaterally to provide inferior and medial support $(\mathrm{C}$ and $\mathrm{D})$. Bilateral inferior capsulorrhaphies were also performed

typically used in the upper pole or superomedial area to thicken tissue, camouflage rippling and minimize the visibility of implant edges. Areas of thinning are marked preoperatively on the breast surface. An extra thick ADM is commonly used and the size will depend on the area being addressed. An access incision remote to the area of thinned tissue is chosen and the pre-existing implant is removed via a capsulotomy. ADM is then placed on the undersurface of the capsule in the area of thinning (or in contact with the neopocket if a site change is being performed), set on appropriate stretch and parachuted into the pocket using marionette sutures as described in the section on symmastia correction. Sutures may be used to suture the ADM to the surrounding tissue; however, if the tissue is very thin, marionette sutures alone may be used to hold the ADM in place and these are placed over bolster sutures on the skin surface and removed on postoperative day 7 .
Use in correction of inferior implant malposition

Many patients with skin laxity will develop inferior displacement of the implant over time. This is more common in patients with large implants in the subglandular plane. Over time, the inferior pole may stretch, increasing the distance from the nipple to the inframammary fold creating what is typically termed 'bottoming out'. In most patients, a site change, placement of a new implant (possibly smaller and/or textured) as well as an overlying mastopexy will be successful. However, in some patients, tissue laxity will lead to recurrence of the problem. In these patients, placement of an ADM inferiorly may bolster the implant and provide long-lasting lower pole soft tissue support.

In a patient with pre-existing large subglandular implants that have displaced inferiorly, site change to a subpectoral pocket is generally employed. If the implant is in the subpectoral plane, a neosubpectoral pocket can be used or a total capsulectomy can be performed. If a Wise 


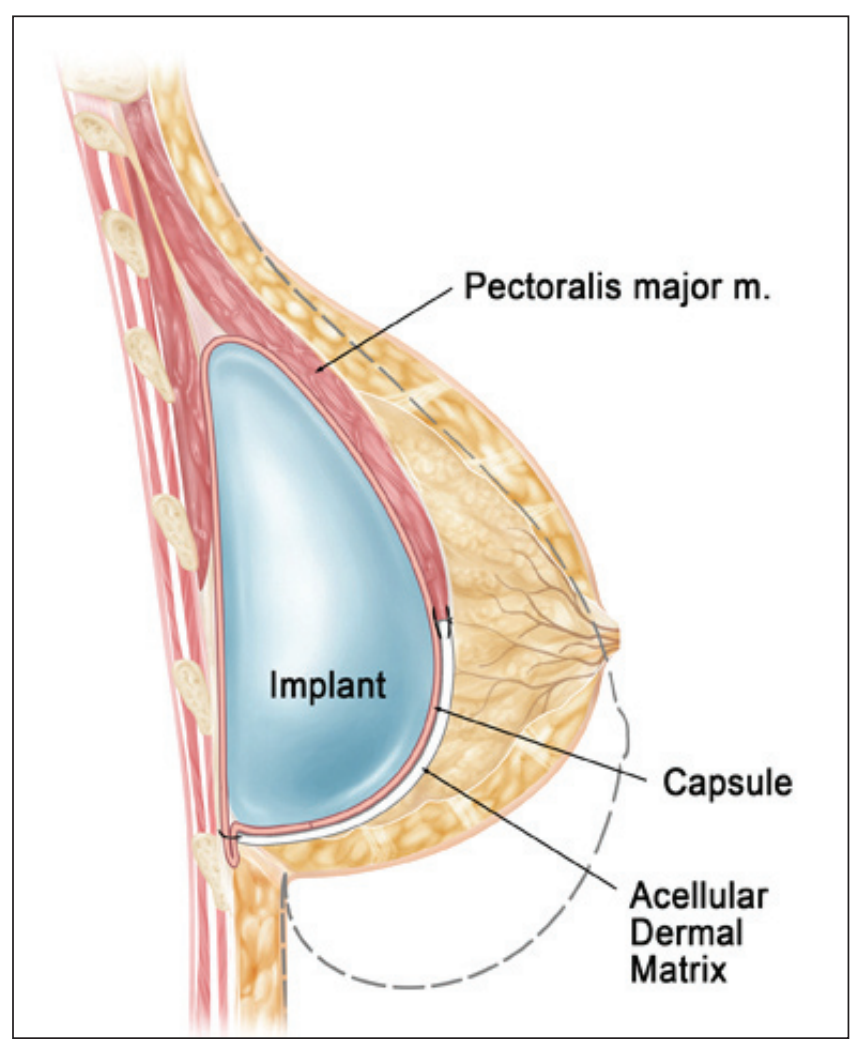

Figure 13) Correction of bottoming-out with acellular dermal matrix. m Muscle

pattern mastopexy is chosen, it is marked preoperatively with the level of the inframammary fold chosen to approximate the dimensions of the new implant (in general, adhering to an $8 \mathrm{~cm}$ nipple-to-inframammary fold distance). An incision is made within the confines of the planned mastopexy markings and dissection proceeds to the level of the capsule. A neosubpectoral pocket is created and the pre-existing implant is removed. If the previous implant was subglandular, the subglandular pocket may be closed with plication sutures. If the previous implant was subpectoral, the previous pocket is similarly closed.

The inferior edge of the pectoral muscle is defined. Marionette sutures are placed medially and laterally to position the ADM as it is parachuted into the pocket. 2-0 Vicryl and running 2-0 PDS are then used to suture the ADM along the inferior and lateral pectoralis major. A temporary sizer is placed and the ADM is draped across its anterior surface and tacked medially and laterally to the chest wall using 2-0 Vicryl. The final implant is then placed and the remaining closure is performed using running 2-0 PDS along the inferior chest wall. Drains are placed and the mastopexy portion of the procedure is then performed (Figures 12A to 12D: use in inferior implant displacement after breast reconstruction).

If a site change is not performed, an inferior capsulorrhaphy can be performed first (Figure 13) and the ADM can then be placed as described above. Alternatively the 'gutter technique' (Figure 14) places ADM over the capsulorrhaphy suture line for reinforcement of the repair (58).

\section{Use in capsular contracture}

The Allergan and Core Study reported a 20.4\% capsular contracture rate after revision of augmentation at seven years. This means that a significant proportion of aesthetic surgery patients will require more than one revision surgery, which imparts significant cost to the patient. There is evidence to suggest that ADMs may resist encapsulation. As we have discussed above, the host may not recognize ADM as foreign, leading to reduced numbers of myofibroblasts and inflammatory cytokines at the ADM/host interface (59-61). This

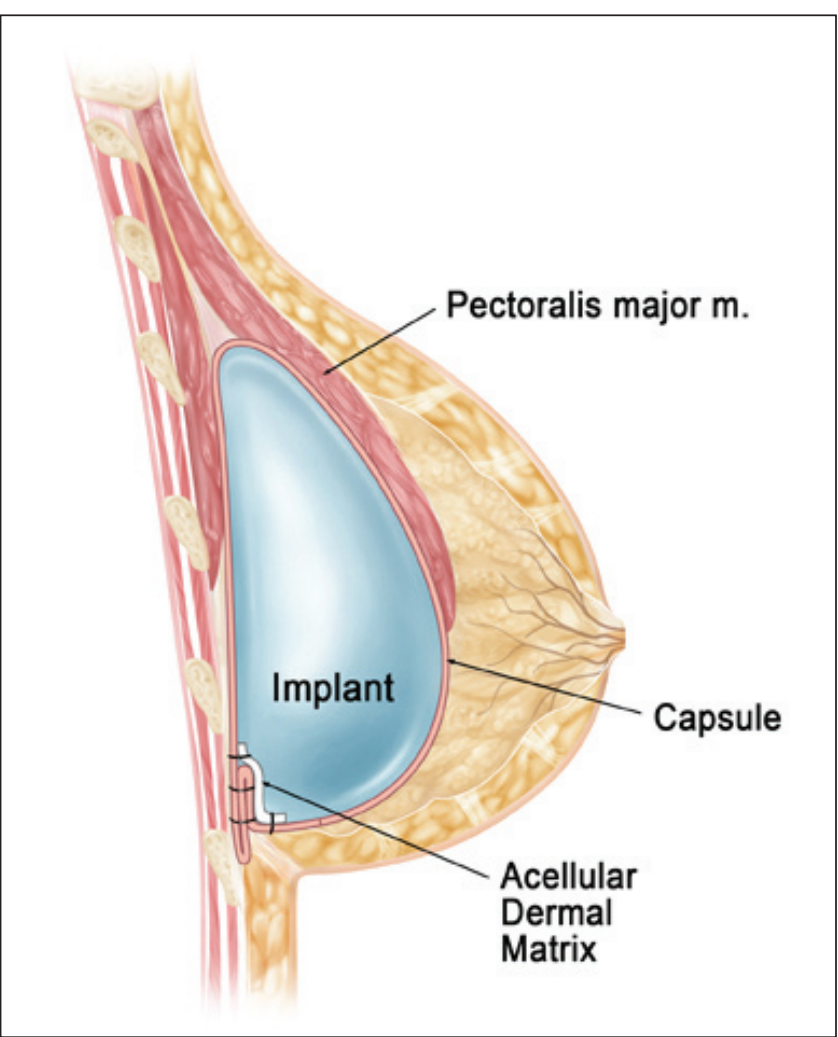

Figure 14) Correction of bottoming out with acellular dermal matrix using the 'gutter' technique. $m$ Muscle

may be reflected clinically by reduced capsular contracture rates. An eight-year review of direct-to-implant reconstruction in 260 patients (28) revealed a very low capsular contracture rate of $0.4 \%$ at 29 months, lending support to the theory that ADM may produce a 'break' or 'firewall' in capsular formation, which would in turn decrease the formation of contracture.

ADM may be used at the primary aesthetic implant surgery or during revisionary surgery. Due to cost, ADMs are generally reserved for treatment of capsular contracture once it has occurred and are incorporated at the revision surgery. In most cases, a total capsulectomy with site change to the subpectoral plane will be chosen. The ADM is placed from the inferior pectoralis muscle, around the surface of the inferior implant and sutured to the chest wall as described in the section 'Use in correction of inferior implant malposition'. A more detailed review of ADMs and capsular contracture follows.

\section{ADMs AND CAPSULAR CONTRACTURE}

Capsular contracture is one of the most common complications in aesthetic and reconstructive breast surgery, with a rate reported to be between $1 \%$ and $20 \%$ for primary breast augmentation (62-64), and much higher for revision augmentation or reconstructive surgery. The cumulative risk for capsular contracture after reconstruction has been reported to be $12 \%$ at one year postoperatively, rising to $30 \%$ at five years postoperatively (65). Several early series of breast reconstruction with ADMs were noted to have a lower than anticipated rate of capsular contracture $(29,47,66)$.

The etiology of capsular contracture remains unknown. However, variables believed to contribute to its development include bacterial colonization or subclinical infection, contaminants around the implant, shear forces acting on the tissues, and seroma or hematoma at the time of implantation (67-69). All of these variables could act through the common pathway of inflammation to increase the deposition of collagen around an implant, with myofibroblasts potentially involved. Prantl et al (70) demonstrated a trend toward a correlation 
between severity of capsular contracture and degree of inflammation. The authors also noted, as have many others, the presence of synovial cell metaplasia in the capsule of $62.5 \%$ of patients.

The scientific data on ADMs and capsule formation suggests that the clinical experience of lower capsular contracture is related to the reduced inflammatory environment in the presence of ADM. Orenstein et al (71) demonstrated that AlloDerm was able to significantly inhibit the production of interleukin (IL)-1, IL-6, IL-8 and vascular endothelial growth factor when placed in contact with peripheral blood monocytes in vitro. As mentioned previously, Komorowska-Timek et al (59) demonstrated that ADMs reduced radiation-induced capsule formation in a rat model. At 10 weeks in a primate model, AlloDerm was found to completely inhibit capsule formation and significantly reduce myofibroblast activity. Biopsies of human breast capsules and associated ADM at the time of implant exchange have demonstrated decreased capsular fibrosis and fibroblast cellularity relative to controls (72).

The current clinical and experimental evidence regarding ADMs and capsular contracture is very encouraging. However, the low rates of capsular contracture seen with ADMs must be assessed in the context of low numbers of patients and short follow-up relative to the overall rates of capsular contracture historically. In addition, the cost of ADMs must be considered relative to the fairly good results described in the past with other revisionary techniques such as implant and pocket exchange, and total capsulectomy (73).

\section{ADMs AND RADIATION}

Indications for adjuvant radiotherapy have expanded recently, with radiotherapy recommended for a single positive lymph node in many centres (74). As a result, there are increasing numbers of breast reconstruction patients who have received previous radiation or who require radiotherapy postoperatively. Many authors have reported poor outcomes with nonautologous reconstruction in the setting of radiation, with higher rates of capsular contracture, infection, revision rates and reconstructive failure (75-77). There is a perception that reconstructions involving ADMs do better in the setting of radiation despite the absence of prospective clinical trials demonstrating such. The literature on this issue is somewhat contradictory. Multiple authors have reported retrospective series and included outcomes of ADM reconstructions with either pre- or postoperative radiation $(26,50,78)$. Colwell et al (26) reported that there were no statistical differences in complication rates in radiated versus nonradiated patients. Kobraei et al (78), however, reported that postoperative radiation was the only variable that had statistical significance as a risk factor for implant loss in all patients regardless of whether an ADM was used, albeit with a small number of patients.

Experimentally, results consistently demonstrate the ability of ADMs to become incorporated and vascularized in either a radiated field or with postimplantation radiation $(79,80)$. Komorowska-Timek and Gurtner (34) specifically studied the effect of radiation on implants wrapped in ADM in an animal model. These authors found decreased inflammation and pseudoepithelium formation in the presence of ADM and noted the ability of the ADM to incorporate into the host tissue despite the radiation. A lack of clarity surrounding the role of ADMs in the setting of either pre- or postimplantation radiation exists. Resolution of this question will require ongoing clinical studies.

\section{COST IMPLICATIONS}

To establish the cost-benefit of a new medical procedure compared with the traditional approach, the probabilities of all possible outcomes must be known. Pooling results from multiple randomized controlled trials (RCTs) would yield the best estimate for outcomes; however, RCTs comparing surgical interventions are rare. When assessing the outcomes of breast surgery with and without the use of ADMs, current data are available only from observational studies.

The most comprehensive type of economic analysis is the costeffectiveness analysis. This generates cost per quality-adjusted life year (QALY) in which a QALY is a value determined by the number of extended life years in a particular utility state attributable to a particular intervention. A utility state is the preference of an individual or society for a particular health state. Utility states for patients living post-breast reconstruction performed with and without ADMs are currently not available and, therefore, formal cost-effectiveness analysis is not possible.

The most common type of economic assessment used when utility states are unavailable is cost minimization. This type of analysis is used to compare two alternatives that have comparable outcomes but different costs, and cost in dollars is the only reported metric (81). Cost minimization analysis is inherently dependent on the probabilities of the outcomes used in the analysis. Thus, this type of analysis can only be used as a guide if the outcome probabilities are estimated from observational data.

In the Canadian universal health care setting, reduction of costs is a priority. If a particular surgical technique is less expensive than an alternative and has comparable outcomes, this technique will be adopted. This is especially important when considering implementation of an expensive new product (ie, ADMs). Direct-to-implant breast reconstruction is the only situation in which a cost savings might be expected because a second stage of surgery is eliminated in the ideal patient.

In 2011, Jansen and Macadam (82) published a cost-minimization analysis comparing direct-to-implant reconstruction using ADM with the traditional two-stage approach without ADM. Probabilities for eight clinically important outcomes (no complication, capsular contracture, seroma, infection, hematoma, implant exposure with loss, implant exposure with salvage and mastectomy flap necrosis) were estimated for direct-to-implant reconstruction with ADM and twostage reconstruction without ADM from a previous systematic review (83). Using a decision analytic model, the cost of direct-to-implant reconstruction with $\mathrm{ADM}$ was estimated to be $\$ 10,734$, whereas the traditional two-stage approach was estimated to cost $\$ 11,251$. Using the online calculator published with this article, we can revise these initial estimates to $\$ 11,072$ versus $\$ 15,049$, respectively, when the weighted values (direct-to-implant, Table 1) and Allergan and Mentor Core Study outcomes (two-stage without ADM, Table 2) are inputted (Figure 15). Cost savings result from elimination of the second stage of surgery and from the lower revision rate estimated for direct-toimplant reconstruction. Current rates of revision are estimated using observational studies with short-term follow-up. Studies with longterm follow-up are required to make more accurate comparisons. These costs are based on a Canadian health care single-payer system and, therefore, cannot be extrapolated to American institutions.

De Blacam et al (84) performed a similar cost-minimization analysis that was published in 2011. This study compared tissue expander/ implant reconstruction, tissue expander/implant with ADM and directto-implant reconstruction with ADM in an American health care model. Using probabilities for five outcomes states estimated from systematic review (no complication, cellulitis, seroma, skin necrosis and implant removal), the authors found the expected cost of tissue expander/implant reconstruction to be $\$ 10,934$ (18), that of tissue expander/implant with ADM to be $\$ 11,255$ (78) and direct-toimplant reconstruction to be $\$ 5,423.02$. Notable differences of this cost analysis when compared with the Canadian study were the inputted cost of ADM at just $\$ 321$ (Medicare fee), and significant differences in hospital fees and surgical fees between two stage and one-stage reconstruction. In the Canadian study, equivalent hospital fees were estimated for each technique, whereas De Blacam et al estimated a $\$ 3000$ difference as well as a $\$ 2000$ difference in surgical fees. Jansen and Macadam (82) estimated a $\$ 500$ difference in surgical fees between the two procedures. This highlights the differences in costs across different health care systems.

The cost-benefit analysis of ADMs in revisional aesthetic surgery is more complicated. If use of an ADM at an initial revisionary surgery decreases the number of further revision surgeries required, this may impart a cost savings to the patient. The two most recent studies published on this topic by Maxwell and Gabriel (52) and Spear et al (58) 


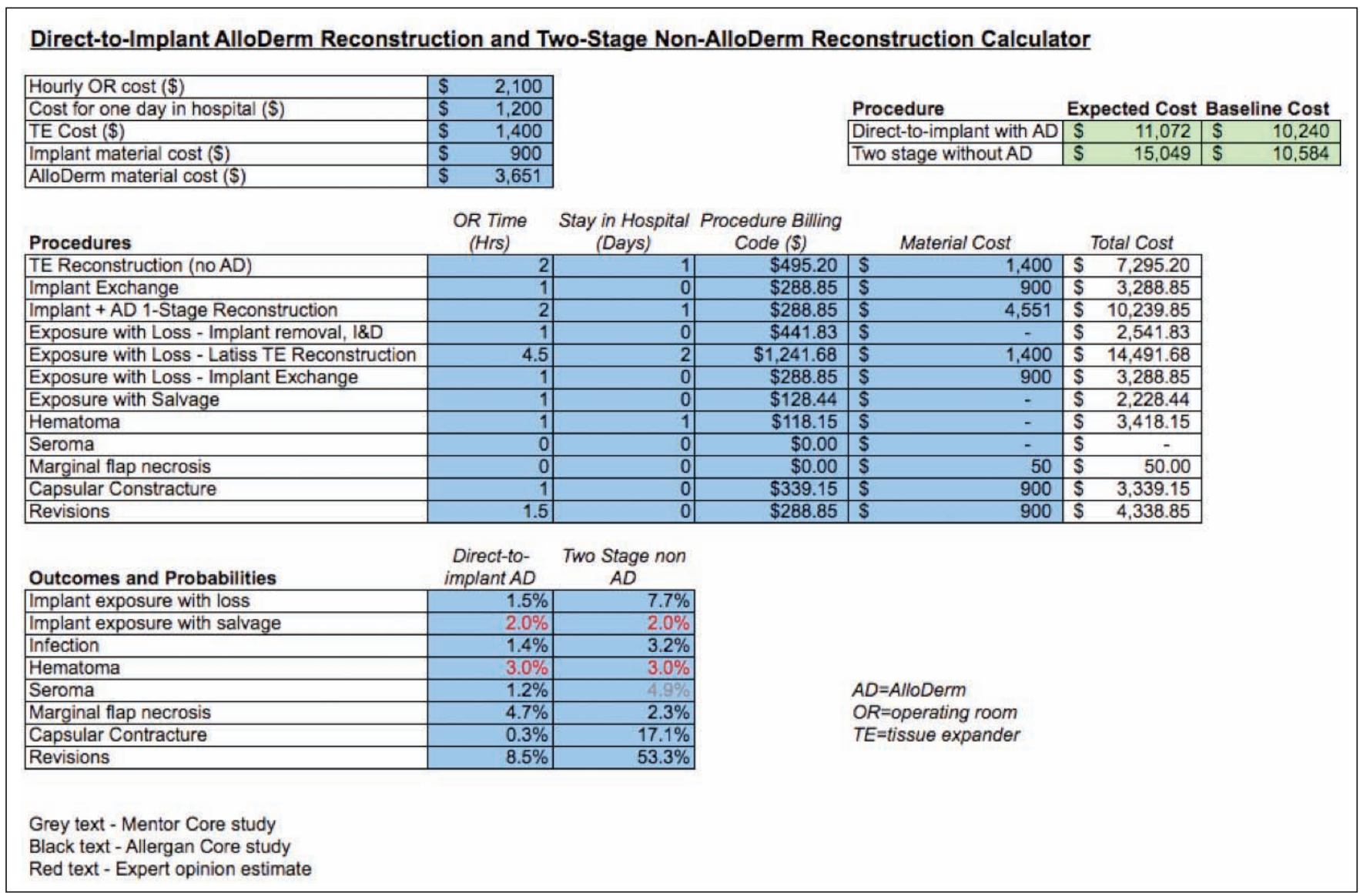

Figure 15) Direct-to-implant AlloDerm (AD, LifeCell Corporation, USA) reconstruction and two-stage non-AD reconstruction calculator. Upper right box with costs in green show expected costs for direct-to-implant (one stage) versus traditional two-stage reconstruction after inputting weighted averages for directto-implant outcomes from Table 1 and the Core Study outcomes for two-stage, non-AD outcomes from Table 2. Outcome probability for exposure with salvage and hematoma probability estimated by expert opinion

show relatively low rates of secondary revision surgery if ADM is used at the first revisionary surgery. Maxwell and Gabriel reported on a series of 78 patients undergoing various aesthetic revisionary procedures with ADM and noted that only two (2.6\%) patients required subsequent surgery with approximately 12 months average follow-up. Spear et al (58) followed 52 patients that had ADM placed at the initial aesthetic surgery or at the first revision surgery and reported three patients (5.8\%) requiring further revisional surgery after nine months follow-up. These numbers compare favourably with the Allergan seven-year Core Study, which found a $40.5 \%$ secondary revision rate after an initial revision following breast augmentation surgery (32).

Further studies with longer term follow-up need to be performed to make conclusive statements about the cost-benefit of ADM use in revisionary aesthetic breast surgery.

\section{CONCLUSION}

Over the past decade, the role of ADMs in reconstructive breast surgery and, to a lesser degree, aesthetic breast surgery, has become well established. Clinical use has expanded greatly, particularly over the past few years and the literature documenting this use has increased in a commensurate fashion. Numerous studies have confirmed the safety of ADMs with complication rates that are comparable with similar procedures without ADM in a variety of clinical applications. It remains unclear whether there are differences in outcomes between the different ADM products currently available on the market.

The literature suggests many potential advantages of ADMs in reconstructive breast surgery; however, to date, the majority of studies are retrospective case series. There are currently two ongoing, prospective
RCTs in breast reconstruction: McCarthy et al at Memorial Sloan Kettering (New York, USA) comparing two-stage ADM-based reconstruction to traditional non-ADM two-stage reconstruction, and Zhong et al at the University of Toronto (Toronto, Ontario) comparing direct-to-implant reconstruction with ADM with traditional two-stage non-ADM reconstruction. These studies will be helpful in determining the role of ADMs in breast reconstruction.

In aesthetic breast surgery, there are several clinical scenarios in which ADMs seem to be useful and may improve outcomes compared with traditional techniques. However, there are no long-term studies or well-designed comparisons that prove the superiority of ADMs in this area. Similarly, there is encouraging evidence that ADMs may be protective in the setting of adjunctive radiotherapy and that ADMs may have a role to play in the treatment or prevention of capsular contracture. The evidence to date, however, is not adequate to make use of ADMs standard of care in these areas and cost remains a barrier for routine use in aesthetic breast surgery.

ADM-assisted breast surgery continues to evolve. Principal advantages include the potential enhancement of cosmesis in breast reconstruction, amelioration of late or irradiation-induced contracture, improved long-term correction of complications following aesthetic revisionary surgery and cost-savings imparted by the direct-to-implant breast reconstruction model. Ongoing well-designed studies are required to clearly define which patient populations and clinical cases will truly benefit from this technology.

DISCLOSURE: Dr Lennox is a speaker for Lifecell. No funding was obtained for the preparation of this article. 


\section{REFERENCES}

1. Wainright DJ. The use of an allograft dermal matrix (AlloDerm) in the management of full thickness burns. Burns 1995:21:243-8.

2. Tal H. Subgingival acellular dermal matrix allograft for the treatment of gingival recession: A case report. J Periodontal 1999; 70:1118-24.

3. Shorr N, Perry JD, Hoenig J, et al. The safety and applications of acellular human dermal allograft in ophthalmic plastic and reconstructive surgery: A preliminary report. Ophthal Plast Reconstr Surg 2000;16:223-30.

4. Sclafani AP, Romo T III, Jacono AA, et al. Evaluation of acellular dermal graft in sheet (AlloDerm) and injectable (micronized AlloDerm) forms for soft tissue augmentation. Clinical observations and histlogical analysis. Arch Facial Plastic Surg 2000;2:120-36.

5. Buinewicz B, Rosen B. Acellular cadaveric dermis (AlloDerm): A new alternative for abdominal hernia repair. Ann Plast Surg 2004;52:188-94.

6. An G, Walter RJ, Nagy K. Closure of abdominal wall defects using acellular dermal matrix. J Trauma 2004;56:1266-75.

7. Terino EO. Alloderm acellular dermal graft: Applications in aesthetic soft-tissue augmentation. Clin Plast Surg 2001;28:83-99.

8. Chaplin JM, Constantino PD, Wolpoe ME et al. Use of an acellular dermal allograft for dural replacement: An experimental study. Neurosurgery 1999;16:196-201.

9. Duncan DI. Correction of implant rippling using allograft dermis. Aesth Surg J 2001;21:81-4.

10. Baxter RA. Intracapsular allogenic dermal grafts for breast implantrelated problems. Plast Reconstr Surg 2003;112;1692-6.

11. Breuing KH, Warren SM. Immediate bilateral breast reconstruction with implants and inferolateral AlloDerm slings. Ann Plast Surg 2005;55:232-9.

12. Bindingnavele V, Gaon M, Ota KS, et al. Use of acellular cadaveric dermis and tissue expansion in postmastectomy breast reconstruction. J Plast Reconstr Aesthet Surg 2007;60:1214-8.

13. Lifecell Corporation (Internal data).

14. Badylak, SF, Freytes DO, Gilbert TW. Extracellular matrix as a biological scaffold material: Structure and function. Acta Biomaterialia 2009;5:1-13.

15. Hafeez YM, Zuki AB, Yusof $N$ et al. Effect of freeze-drying and gamma irradiation on biomechanical properties of bovine pericardium. Cell Tissue Bank 2005;6:85-9.

16. Xu H, Wan H, Sandor M, et al. Host response to human acellular dermal matrix transplantation in a primate model of abdominal wall repair. Tissue Eng Part A. 2008;14:2009-19.

17. Sandor M, Xu H, Connor J, et al. Host response to implanted porcine derived biologic materials in a primate model of abdominal wall repair. Tissue Eng 2008: Part A 14:2021.

18. AlloDerm Regenerative Tissue Matrix: Instructions For Use. $<$ www.lifecell.com/downloads/LC Alloderm114 IFU B T4.pdf> (Accessed March, 2012).

19. FlexHD and FlesHD Diamond: Instructions for Use. <www.mtf.org/ documents/PI_-31 Rev 12.pdf> (Accessed March 3, 2012).

20. DermaMatrix: Instructions for Use. <www.mtf.org/documents/PI_23_Rev_9.pdf $>$ (Accessed March 2012).

21. AlloMax: Technique Guide. <www.davol.com/default/assets/File/ MMAMBRTG.pdf $>$ (Accessed March 2012).

22. SurgiMend: Safety and Handling. <www.teibio.com/Literature/ SurgiMend/Product\%20Information/Safety\%20\&\%20Handling\%20 -\%20SurgiMend\%201.0-4.0/PN\%20606-999-023v02.pdf> (Accessed March 2012).

23. Strattice Implantation Instructions. <www.lifecell.com/downloads/ StratticeIFU_T11.pdf $>$ (Accessed March 2012).

24. Ashikari RH, Ashikari AY, Kelemen PR, et al. Subcutaneous mastectomy and immediate reconstruction for prevention of breast cancer for high-risk patients. Breast Cancer 2008;15:185-191.

25. Austen WG, Hartzell TL, Hertl MC. A simplified technique for single stage breast reconstruction. Poster presented at: $2006 \mathrm{New}$ England Surgical Society Meeting; September 16, 2006; Groton, Connecticut, 2001.

26. Colwell AS, Damjanovic B, Zahedi B et al. Retrospective review of 331 consecutive immediate single-stage implant reconstructions with acellular dermal matrix: Indications, complications, trends and costs. Plat Reconstr Surg 2011;128:1170-8.

27. Gamboa-Bobadilla GM. Implant breast reconstruction using acellular dermal matrix. Ann Plast Surg 2006;56:22-5.
28. Salzberg CA, Ashikari AY, Koch RM et al. An 8-year experience of direct-to-implant immediate breast reconstruction using human acellular dermal matrix (AlloDerm). Plast Reconstr Surg 2011;127:514-24.

29. Salzberg CA. Nonexpansive immediate breast reconstruction using human acellular tissue matrix graft (AlloDerm). Ann Plast Surg 2006;57:1-5.

30. Topol BM, Dalton EF, Ponn T et al. Immediate single-stage breast reconstruction using implants and human acellular dermal tissue matrix with adjustment of the lower pole of the breast to reduce unwanted lift. Ann Plast Surg 2008;61:494-9.

31. Zienowicz RJ, Karacaoglu E. Implant-based breast reconstruction with allograft. Plast Reconstr Surg 2007;120:373-81.

32. <www.allergan.com/assets/pdf/L034-03 silicone DFU.pdf> (Accessed March 2012).

33. <www.mentorwwwlc.com/pdf/approved/gel-PIDS.pd> (Accessed March 2012).

34. Komorowska-Timek E, Gurtner GC. Intraoperative perfusion mapping with laser-assisted indocyanine green imaging can predict and prevent complications in immediate breast reconstruction. Plast Reconstr Surg 2010;125:1065-73.

35. Antony AK, McCarthy CM, Cordeiro PG, et al. Acellular human dermis implantation in 153 immediate two-stage tissue expander breast reconstructions: Determining the incidence and significant predictors of complications. Plast Reconstr Surg 2010;125:1606-14.

36. Becker S, Saint-Cyr M, Wong C, et al. AlloDerm versus DermaMatrix in immediate expander-based breast reconstruction: A preliminary comparison of complication profiles and material compliance. Plast Reconstr Surg 2009;123:1-6; discussion 107-8.

37. Hanna KR, DeGeorge BR, Mericli AF, et al. Comparison of two types of expander-based reconstruction: Acellular dermal matrixassisted vs total submuscular placement Ann Plast Surg 2011 (Epub ahead of print).

38. Lanier ST, Wang ED, Chen JJ, et al. The effect of acellular dermal matrix use on complication rates in tissue expander/implant breast reconstruction. Ann Plast Surg 2010;64:674-8.

39. Liu AS, Kao HK, Reish RG, et al. Postoperative complications in prosthesis-based breast reconstruction using acellular dermal matrix. Plast Reconstr Surg 2011;127:1755-62.

40. Losken A. Early results using sterilized acellular human dermis (NeoForm) in postmastectomy tissue expander breast reconstruction Plast Reconstr Surg 2006;123:1654-8.

41. Margulies AG, Hochberg J, Kepple J, et al. Total skin-sparing mastectomy without preservation of the nipple-areola complex. Am J Surg. 2005;190:907-12.

42. Nahabedian MY. AlloDerm performance in the setting of prosthetic breast surgery, infection, and irradiation. Plast Reconstr Surg 2009; $124: 1743-53$.

43. Namnoum JD. Expander/implant reconstruction with AlloDerm: Recent experience. Plast Reconstr Surg 2009;124:387-94.

44. Parikh PM, Spear SL, Menon N, et al. Immediate breast reconstruction with tissue expanders and alloderm. Plast Reconstr Surg 2006;118(Suppl 4):18.

45. Preminger BA, McCarthy CM, Hu QY, et al. The influence of AlloDerm on expander dynamics and complications in the setting of immediate tissue expander/implant reconstruction: A matchedcohort study. Ann Plast Surg 2008;60:510-3.

46. Sbitany H, Sandeen SN, Amalfi AN, et al. Acellular dermisassisted prosthetic breast reconstruction versus complete submuscular coverage: A head-to-head comparison of outcomes. Plast Reconstr Surg 2009;124:1736-40.

47. Spear SL, Parikh PM, Reisin E et al. Acellular dermis-assisted breast reconstruction Aesth Plast Surg 2008;32:418-25.

48. Vardanian AJ, Clayton JL, Roostaeian J, et al. Comparison of implant-based immediate breast reconstruction with and without acellular dermal matrix. Plast Reconstr Surg 2011;128:403e-409e.

49. Kim JYS, Davila AA, Persing S, et al. A meta-analysis of human acellular dermis and submusuclar tissue expander breast reconstruction. Plast Reconstr Surg 2011;129:28-41.

50. Sbitany H, Serletti JM. Acellular dermis-assisted prosthetic breast reconstruction: A systematic and critical review of efficacy and associated morbidity. Plast Reconstr Surg 2011;128:1162-9.

51. Hoppe IC, Yueh JH, Wei CH, et al. Complications following expander/implant breast reconstruction utilizing acellular dermal matrix: A systematic review and meta-analysis. ePlasty 2011;11e40:417-28. 
52. Maxwell GP, Gabriel A. The neopectoral pocket in revisionary breast surgery. Aesthet Surg J 2008;28:463-7.

53. Chasan PE. Breast capsulorrhaphy revisited: A simple technique for complex problems. Plast Reconstr Surg 2005;115:296-301.

54. Spear SL, Dayan JH, West J. The anatomy of revisions after primary breast augmentation: One surgeon's perspective. Clin Plast Surg 2009;36:157-65.

55. Spear SL, Bogue DP, Thomassen JM. Synmastia after breast augmentation. Plast Reconstr Surg 2006;118(7 Suppl):168S-171S.

56. Maxwell GP, Gabriel A. Use of acellular dermal matrix in revisionary aesthetic breast surgery. Aesth Surg J 2009;29:485-93.

57. Maxwell GP, Gabriel A. Acellular dermal matrix in aesthetic revisionary breast surgery. Aesth Surg J 2011;31:65s-76s.

58. Spear S, Seruya M, Clemens MW, et al. Acellular dermal matrix for the treatment and prevention of implant-associated breast deformities. Plast Reconstr Surg 2011;127:1047-58.

59. Stump A, Holton LH, Connor J, et al. The use of acellular dermal matrix to prevent capsule formation around implants in a primate model. Plast Reconstr Surg 2009;124:82-91.

60. Uzunismail A, Duman A, Perk C et al. The effects of acellular dermal allograft $\left(\right.$ AlloDerm $\left.{ }^{\circledR}\right)$ interface on silicone related capsule formation-experimental study. Eur J Surg 2008;31:170-85.

61. Basu CB, Leong M, Hicks, J. Does acellular cadaveric dermis (ACD) affect breast implant capsule formation in reconstructive breast surgery? A histopathologic comparison of breast capsule and ACD. Plast Reconstr Surg 2010;126:1842-7.

62. Spear SL, Murphy DK, Slicton et al. Inamed silicone breast implant core study results at 6 years. Plast Reconstr Surg 2007;120(7 Suppl 1):8S-16S.

63. Cunningham B, McCue J. Safety and effectiveness of Mentor's MemoryGel implants at 6 years. Aesthetic Plast Surg 2009;33:440-4.

64. Tebbetts JB. Achieving a zero percent reoperation rate at 3 years in a 50 consecutive case augmentation mammoplasty pre market approval study. Plast Reconst Surg 2006;118:1453-7.

65. Handel N, Cordray T, Gutierrez J et al. A long term study of outcomes, complications, and patient satisfaction with breast implants. Plast Reconstr Surg 2006;117:757-67.

66. Breuing K, Colwell AL. Inferolateral AlloDerm hammock for imlant coverage in breast reconstruction. Ann Plast Surg 2007;59:250-5.

67. Adams WP. Capsular contracture: What is it? What Causes it? How can it be prevented or managed? Clin Plast Surg 2009;36:1:119-26.

68. Wong CH, Samuel M, Tan BK et al. Capsular contracture in subglandular breast augmentation with textured versus smooth breast implants: A systematic review. Plast Reconstr Surg 2006;118:1224-36.
69. Henriksen T, Trine F, Fryzek JP et al. Surgical intervention and capsular contracture after breast augmentation: A prospective study of risk factors. Ann Plast Surg 2005;54:343-51.

70. Prantl L, Schreml S, Fichtner-Feigl S, et al. Clinical and morphological conditions in capsular contracture formed around silicone breast implants. Plast Reconstr Surg 2007;120:275-84.

71. Orenstein SB, Qiao Y, Kaur M et al. Human monocyte activation by biologic and biodegradable meshes in vitro. Surg Endosc 2010;24:805-11. Epub 2009 Aug 21.

72. Basu CB, Leong M, Hicks J. Acellular cadaveric dermis decreases the inflammatory response in capsule formation in reconstructive breast surgery. Plast Reconstr Surg 2010:126:1842-7.

73. Spear S, Carter ME, Ganz JE. The correction of capsular contracture by conversion to "dual-plane" positioning: Technique and outcomes. Plast Reconstr Surg 2006;118(Suppl):103S-113S.

74. Wei ES, Lesperance M, Speers CH et al. Increased use of regional radiotherapy is associated with improved outcome in a population based cohort of women with breast cancer with 1-3 positive nodes. Radiother Oncol 2010;97:301-6.

75. Kronowitz SJ, Robb GL. Breast reconstruction with postmastectomy radiation therapy: Current issues. Plast Reconstr Surg 2004;15:950-60.

76. Lee BT, Adesiyun T, Colakoglu S, et al. Postmastectomy radiation therapy and breast reconstruction: An analysis of complication and patient satisfaction. Ann Plast Surg 2009;62:350-4.

77. Spear SL, Oneyewu C. Staged breast reconstruction with saline filled implants in the irradiated breast: Recent trends and therapeutic implications. Plast Reconstr Surg 2000;105:930-42.

78. Kobraei EM, Nimtz J, Wong L et al. Risk factors for adverse outcome following skin sparing mastectomy and immediate prosthetic reconstruction. Plast Reconstr Surg 2012;234e-41e.

79. Dubin MG, Feldman M, Ibrahim HZ et al. Allograft dermal implant (AlloDerm) in a previously irradiated field. Laryngoscope 2000;110:934-7.

80. Ibrhaim HZ, Kwiatkowski TJ, Montone KT et al. Effects of external beam radiation on the allograft dermal implant. Otolaryngol Head Neck Surg 2000;122:189-94.

81. Thoma A, Sprague S, Tandan V. Users' guide to the surgical literature: How to use an article on economic analysis. Can J Surg 2001;44:347-54.

82. Jansen L, Macadam SA. The Use of AlloDerm in postmastectomy alloplastic breast reconstruction: Part II. A cost analysis. Plast Reconstr Surg 2011;127:2245-54.

83. Jansen L, Macadam SA. Theuse of AlloDerm in postmastectomy alloplastic breast reconstruction: Part I. A systematic review. Plast Reconstr Surg 2011;127:2232-44.

84. De Blacam C, Momoh AL, Colakoglu S et al. Cost analysis of implant-based breast reconstruction with acellular dermal matrix. Ann Plast Surg 2011; May 16 (E-Pub ahead of print). 